\begin{tabular}{|l|l|l|l|}
\hline Eiszeitalter u. Gegenwart & $\mathbf{3 1}$ & $\begin{array}{c}135-158 \\
8 \mathrm{Abb} ., 5 \mathrm{Tab} .\end{array}$ & Hannover 1981 \\
\hline
\end{tabular}

\title{
Über die Anwendung der Dendrochronologie in der Moorforschung am Beispiel subfossiler Eichenstämme aus niedersächsischen Mooren
}

\author{
Axel Delorme, Hanns-Hubert Leuschner, Hans-Christoph Höfle \& Jes Tüxen *) \\ Dendrochronology, Quercus, Prehistory (150-350 BC), landform evolution, peat bog, inventory, \\ section, C-14 dating, statistical population, index map.
}

Northwestern German Plain (Borsum, Sieden, Donstorf) Niedersachsen. TK 2909, 3317, 3319

$\mathrm{Kurzf}$ assung: Die jahrringchronologische Auswertung subfossiler Eichenstämme aus drei niedersächsischen Mooren führt zum Nachweis eines im wesentlichen zwischen 350 und $150 \mathrm{v}$. Chr. an den verschiedenen Fundstellen gleichzeitig erfolgten Absterbens von Eichenbeständen. Es ergeben sich Hinweise auf eine Klimaschwankung in dieser Zeit. Die Bildungsgeschichte des Hochmoores bei Sieden läßt sich durch die Aufnahme von Baumfunden in situ in Verbindung mit den zugehörigen Moorprofilen rekonstruieren. Das norddeutsche Jahrringmaterial kann mit der Eisenzeitlichen Auwaldeichenchronologie synchronisiert werden und verlängert diese um gut $200 \mathrm{Jahre}$ zum Älteren.

\section{[Dendrochronology in Research on Bogs: Subfossil Oak Logs from Bogs in Lower Saxony]}

A bstract: The chronological evaluation of the annual rings of subfossil oak trunks from three bogs in Lower Saxony provided evidence for the dying off of the oak groves at three locations at about the same time around 150 to $350 \mathrm{BC}$. There are indications of a climatic change at that time. The development of the high bog near Sieden can be reconstructed on the basis of tree logs found in their original position in the profile of the bog.

The data obtained from annual rings in North Germany can be synchronized with the iron age chronology of riperian forest oaks, extending the chronology 200 years further into the past.

\section{Einleitung}

Baumfunde aus Mooren sind seit langem bekannt. Beim Torfabbau, bei der Moorkultivierung, beim Straßen- und Wasserbau werden in bestimmten Moorgebieten immer wieder Bäume und Baumteile unterschiedlicher Spezies, Dimension und Erhaltung freigelegt. HAYEN hat (1960) derartige Funde aus dem nordwestlichen Niedersachsen genauer beschrieben und gedeutet.

Die dendrochronologische am intensivsten bearbeiteten Holzarten Europas sind die Eichen (Quercus robur und Q. petraea). Sie lassen sich holzanatomisch nicht mit Sicherheit unterscheiden, was für ihre dendrochronologische Auswertung auch nicht notwendig ist. Ihre Vorrangstellung verdanken die Eichen mehreren günstigen Umständen. Sie weisen in diesem Raum eine weite geographische Verbreitung auf und sind in historischer wie prähistorischer Zeit als Bau- und Werkholz bevorzugt verwendet worden. Die dendrochronologische Auswertung von Eichenhölzern wird wesentlich erleichtert durch das hohe natürliche Lebensalter dieser Baumarten; d. h. häufig zur Verfügung stehende lange Jahrringfolgen, durch die hohe Dauerhaftigkeit des Holzes, durch das Fehlen von Jahrringanomalien und durch die vorhandene deutliche Kern-Splintgrenze. So ist erklärlich, war-

*) Anschriften der Verfasser: Dr. A. D e lor m e, H.-H. L e u s ch n e r, Institut für Forstbenutzung der Universität Göttingen, Büsgenweg 4, D-3400 Göttingen. - Dr. J. T ü x e n, Dr. H.-C. Hö fle, Niedersächsisches Landesamt für Bodenforschung, Postfach 5101 53, Stilleweg 2, D-3000 Hannover 51. 
um in Europa die meisten und längsten Stanc'ardchronologien für Eiche aufgebaut worden sind.

Seit einigen Jahren werden in den Jahrringlabors der Universitäten Stuttgart-Hohenheim, Göttingen und Köln subfossile Auwaldeichen ausgewertet, die vor allem beim Kiesabbau in verschiedenen Flußtälern freigelegt werden. Mit diesem Material konnten zahlreiche, z. T. über 1000 Jahre umfassende Teilchronologien aufgebaut werden, die nach Radiokarbondaten bis in das Boreal zurückreichen und als Bausteine für eine absolute Jahrringchronologie des Postglazials dienen (BeCKer 1975, Delorme 1978, Schmidt 1977).

Subfossile Baumfunde aus Mooren sind in Kontinentaleuropa bislang nur vereinzelt Gegenstand dendrochronologischer Untersuchungen gewesen. MUNAUT (1967) untersuchte in den Niederlanden subfossile Kiefern - meist Stubben - aus einem Moor bei Terneuzen und, gemeinsam mit CASPARIE (1971), dem Bourtanger Moor bei Emmen. Es konnten mehrere Gruppen synchroner Hölzer ausgeschieden werden, die verschiedene nach Radiokarbondaten einzuordnende Bewaldungsphasen belegen. Innerhalb der Gruppen ließ sich aus den individuellen Jahrringfolgen ableiten, in welcher Zeit jeweils die Bewaldung in Stillstandsphasen des Moorwachstums erfolgte bzw. der Wald als Folge rascher Wiedervernässung und erneuter Torfbildung abstarb.

Die wenigen in niederländischen Mooren gefundenen Eichenhölzer waren für eine jahrringanalytische Auswertung nicht geeignet.

Eindrucksvolle und in ihrer Art einmalige Ergebnisse erzielte die dendrochronologische Forschung in Nordirland. Subfossile Eichenstämme, die vornehmlich beim Straßenbau in Moorgebieten innerhalb eines Areals von $120 \mathrm{~km}$ in N-S-Richtung und $80 \mathrm{~km}$ in E-W-Richtung freigelegt worden waren, dienten als Material zum Aufbau mehrerer Teilchronologien. Diese belegen - mit einigen Lücken - ein breites Zeitspektrum von etwa 1100 bis 8200 v. h., wobei das längste Teilstück mit 3000 Jahren von 3950 bis 6950 v. h. reicht (PILCHER et al. 1977). Optimale Voraussetzungen machten es den nordirischen Dendrochronologen möglich, aus einer vergleichsweise geringen Zahl von Stämmen lange Chronologien aufzubauen: die unter dem Moor begrabenen Eichen entstammten nicht etwa nur wenigen kurzen Bewaldungsphasen, ihr Absterben verteilte sich vielmehr über lange Zeiträume, wobei Einzelbäume relativ hohe Lebensalter erreichten. Somit standen lange, sich immer wieder überlappende und gleichzeitig verlängernde Jahrringfolgen zur Verfügung.

Angaben über Eichenstämme aus niedersächsischen Mooren finden sich — im Gegensatz zu entsprechenden Funden der Baumart Kiefer - in der Literatur nur vereinzelt. Dies mag erklären, warum sich die Dendrochronologie in Norddeutschland erst vergleichsweise spät der Auswertung derartiger Baumfunde gewidmet hat.

Anlaß für die vorliegende Untersuchung gab das Niedersächsische Landesamt für Bodenforschung in Hannover, das bei geologischen Kartierungsarbeiten im Emsland eine Anzahl von Proben subfossiler Eichenstämme für eine jahrringanalytische Auswertung sicherstellte.

\section{Fundstellen}

\subsection{Borsumer Moor bei Rhede an der Ems}

Bei Kartierungsarbeiten stieß Höfle 1979 auf ausgebaggerte Eichenstämme. Mit der Motorsäge wurden Scheiben aus den Stämmen herausgesägt.

Fundstelle 1 (Abb. 1 und 2)

Bl. 2909 Rhede der TK 25: R 2584690

H 5877620 
Geologisches Profil im Bereich der Fundstelle 1:

bis $0,50 \mathrm{~m}$ Niedermoortorf, tiefgepflügt

- $0,70 \mathrm{~m}$ Erlenbruchwaldtorf

- $0,80 \mathrm{~m}$ Birken-Erlenbruchwaldtorf

- $0,95 \mathrm{~m}$ Holz (vermutlich Birke)

- 1,10 m Feinsand, sehr stark mittelsandig = Flußsand der Jüngeren Ems-Niederterrasse

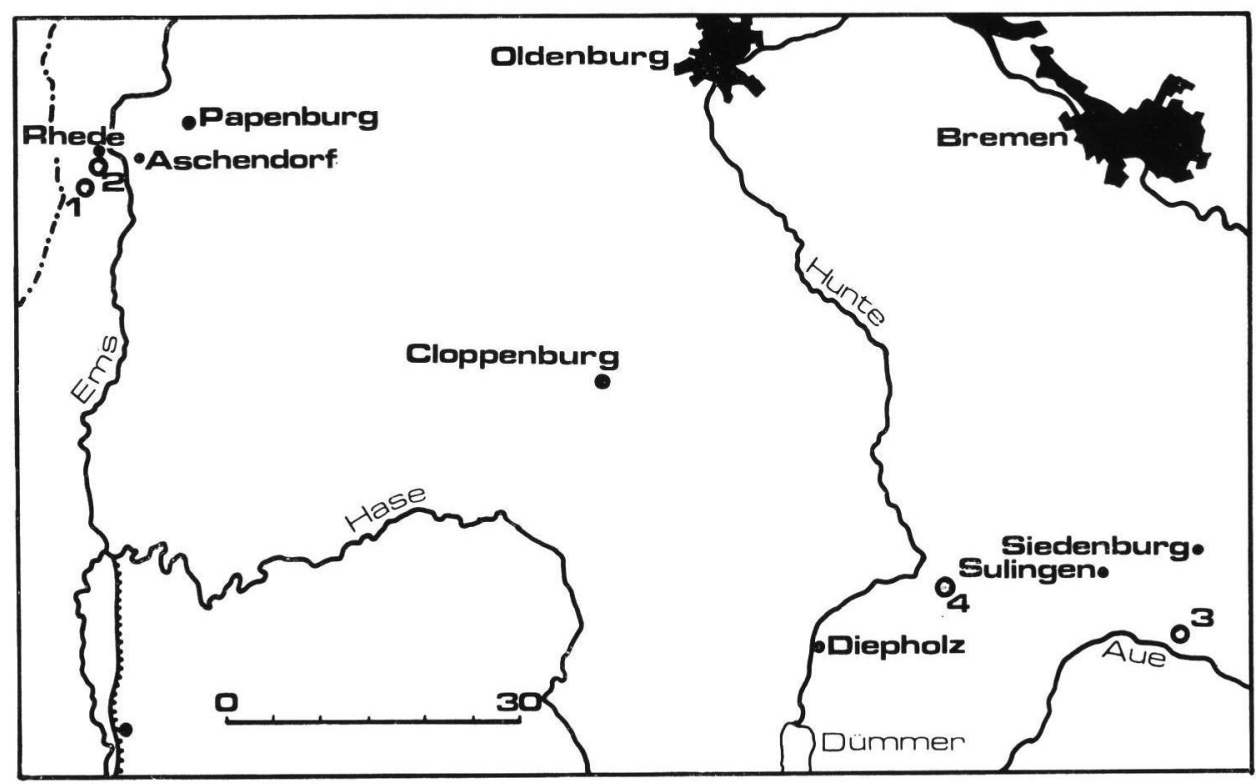

Abb. 1: Lage der untersuchten Moore: Borsumer Moor (1, 2), Hochmoor bei Sieden (3), Donstorfer Moor (4).

Die Eichenstämme waren beim Bau eines 1,50 m tiefen Entwässerungsgrabens aus dem tiefsten Teil der Torfe herausgezogen worden. Der Graben ist etwa $800 \mathrm{~m}$ lang, verläuft in E-W-Richtung und hat an der Fundstelle seinen östlichsten Punkt. Die Bäume waren an verschiedenen Stellen entlang des Grabens herausgebaggert und an der Fundstelle zusammengefahren worden.

F und stelle 2 (Abb. 1 und 2)

Bl. 2909 Rhede der TK 25: R 2586270

H 5879860

Geologisches Profil im Bereich der Fundstelle 2:

bis $0,70 \mathrm{~m}$ Niedermoortorf, sehr stark schluffig, tonig

- $1,10 \mathrm{~m}$ Bruchwaldtorf mit viel Holz

- 1,70 m Feinsand mit Pflanzenhäcksel, Holozän

- 2,00 m Feinsand, Weichsel-Kaltzeit

Die an der Fundstelle 2 liegenden Eichen waren ebenfalls beim Grabenbau von der Torfbasis heraufgeholt worden. Der Graben erstreckt sich von der Fundstelle aus etwa $500 \mathrm{~m}$ nach $\mathrm{W}$. 

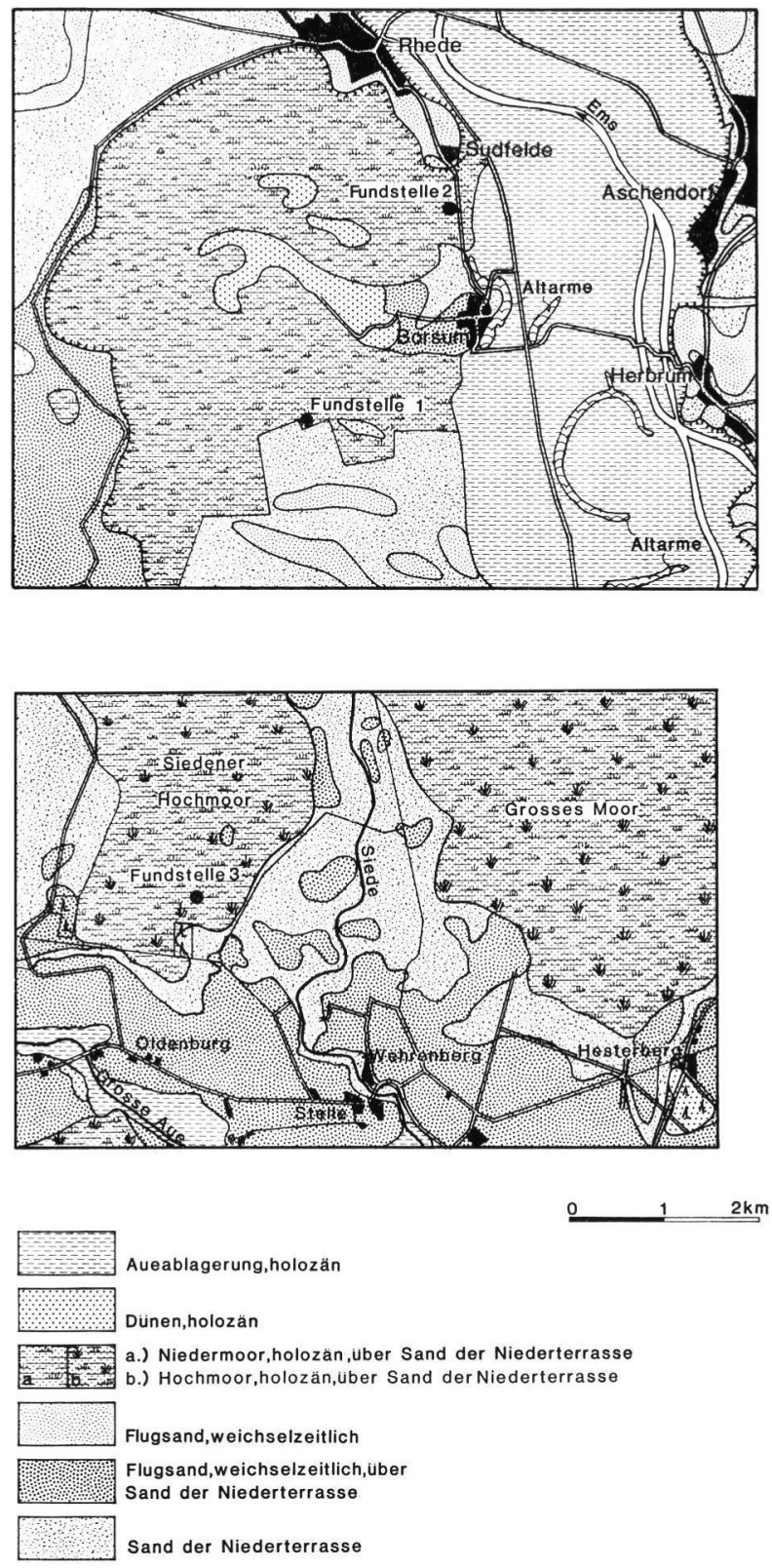

Abb. 2: Geologische Übersicht des Bereiches um das Borsumer Moor (oben) und um das Hochmoor bei Sieden (unten).

Der Niedermoortorf, in dem die Stämme an beiden Fundstellen konserviert wurden, ist auf die Jüngere Niederterrasse der Ems aufgewachsen. Die Oberfläche dieser Terrasse liegt in der näheren Umgebung der Fundpunkte etwa 1,5 bis 3,0 m unter dem Niveau der Alteren Niederterrasse (Terrassenrand-Kammsignatur in Abb. 2). Beide Terrassen sind 
hauptsächlich während der Weichsel-Kaltzeit entstanden. HesemanN (1950) und ThierMANN (1973) haben für den in Nordrhein-Westfalen liegenden Teil des Emsgebietes (mehr als $75 \mathrm{~km}$ südlich) eine Zweiteilung der Niederterrasse mit Niveauunterschieden von bis zu 4 m festgestellt. Durch die Kartierungen des Niedersächsischen Landesamtes für Bodenforschung für die geologische Übersichtskarte $1: 200000$ (Höfle, MERKT \& MEYer in den Jahren 1976 und 1977) konnte die Zweiteilung der Ems-Niederterrasse bis auf die Höhe von Papenburg verfolgt werden. Dann taucht die Jüngere Niederterrasse unter die holozänen Ablagerungen der Ems ab und ist morphologisch nicht mehr erkennbar.

Petrographisch sind die beiden Terrassen auf der Höhe von Rhede/Papenburg nicht unterscheidbar. Deutliche Unterschiede gibt es in der Flugsandbedeckung. Während die Flugsande auf der älteren Niederterrasse meist im Millimeterbereich feingeschichtet sind und relativ häufig den allerödzeitlichen Usselo-Horizont aufweisen, ist bei den Flugsanden auf der Jüngeren Niederterrasse die Schichtung deutlich schlechter ausgeprägt und es fehlt die allerödzeitliche Bodenbildung (Thiermann 1973, Meyer 1977). Im Gebiet um Rheine nimmt Thiermann (1973) die Einschneidung der Ems in die ältere Niederterrasse während der Jüngeren Dryaszeit (= Jüngere Tundrenzeit) an.

H. MüLleR (1956) hat in Altwasserarmen, die in die Jüngere Niederterrasse der Ems bei Walchum eingesenkt waren, und deren Sohle etwa 1,5 m über der Oberfläche der heutigen Ems-Aue liegt, Pollenanalysen an Torfen durchgeführt. Das Torfwachstum begann dort im jüngeren Atlantikum (etwa 3000-2000 v. Chr.). Die Eintiefung und Auffüllung (?Erosionsterrasse) der Jüngeren Niederterrasse müssen daher älter sein.

Über den Beginn der Bewaldung der Terrasse sind z. Zt. keine Aussagen möglich. Erst als eine zunehmende Vernässung und das damit verbundene Torfwachstum für die Konservierung absterbender Bäume sorgten, setzen die Datierungsmöglichkeiten ein.

Zwischen Dorsum und Rhede haben einige Bohrungen unter dem Niedermoortorf und auf den Ablagerungen der Jüngeren Niederterrasse Feinsande mit viel Pflanzenhäcksel und Holzresten angetroffen. Das bedeutet, daß dort vor Beginn des Torfwachstums die Jüngere Niederterrasse im Holozän verschiedentlich überflutet worden ist. An der Fundstelle 2 ist der Torf im oberen Bereich sehr stark mit Schluff und Ton durchsetzt. Dabei handelt es sich um Material, das vor der Eindeichung der Ems in Flußnähe bei Überflutungen eingelagert wurde (Auelehm!).

\subsection{Hochmoor bei Sieden}

Fund ste 11 e 3 (Abb. 1 und 3)

Bl. 3319 Siedenburg der TK 25

Mittelpunkt des Fundgebietes: R 3493660

H 5832360

Nach Hinweisen des Torfwerkes A.G. Meiners in Borstel ${ }^{1}$ ) stieß LeUsChNER auf zahlreiche im Torf liegende Eichenstämme. Die jahrringchronologische Auswertung von 14 geschnittenen Eichenstammscheiben ließ eine weitere Probenentnahme bei gleichzeitiger genauer Erfassung der Fundumstände lohnend erscheinen.

Im September 1980 wurde dann die in Abb. 2 dargestellte Fundstelle an der Südgrenze des Abbaufeldes nördlich der Siedlung Vogtei untersucht. Hinweise auf die an der Basis des Hochmoortorfes liegenden Bäume ergaben sich in den abgetorften Streifen (Pütten) und entlang eines Entwässerungsgrabens. Da die Torfstechmaschine beim Auftreffen

1) Wir sind Herrn Lieckweg vom Torfwerk für die Ermöglichung der Geländearbeiten sehr dankbar. 
auf einen Stamm diesen überspringt und eine entsprechende Erhöhung in der Pütte hinterläßt, wurden vor allem derartige Geländeunebenheiten auf darunter verborgene Baumstämme hin untersucht.

Durch Abtasten der von Torf bedeckten Stammteile wurde die Stammlänge sowie die Lage des Stammfußes ermittelt; letzteres ist unerläßlich für das Bestimmen der Fallrichtung.

Nachdem auf diese Weise noch einmal 26 Stämme erfaßt wurden, standen insgesamt 40 Hölzer auf dem Siedener Hochmoor zur Verfügung. Von den Bäumen Nr. 17 bis 43 wurden durch das Niedersächsische Landesamt für Bodenforschung die genaue Lage und die Höhe gemessen. Die Lage ist in Abb. 7 dargestellt. Die Hoch- und Rechtswerte sowie die Höhe der Oberkanten zu NN sind in Tabelle 1 aufgeführt.

Tabelle 1: Fundkoordinaten und Absterbedaten subfossiler Baumstämme aus dem Siedener Hochmoor

\begin{tabular}{|c|c|c|c|c|c|}
\hline Stamm Nr. & Baumart & $r-$ Wert & h - Wert & Oberkante zu NN & Absterbejahr \\
\hline 17 & Eiche & 3493677 & 5832225 & $+32.15 \mathrm{~m}$ & nach 2148 v. \\
\hline 18 & Eiche & 3493659 & 5832235 & $+32.18 \mathrm{~m}$ & um 2161 v. h. \\
\hline 19 & Eiche & 3493703 & 5832280 & $+32.04 \mathrm{~m}$ & um 2288 v. h. \\
\hline 20 & Erle & 3493705 & 5832344 & $+31.94 \mathrm{~m}$ & \\
\hline 22 & Eiche & 3493714 & 5832385 & $+32.25 \mathrm{~m}$ & nach 2151 v. h. \\
\hline 23 & Eiche & 3493703 & 5832390 & $+32.08 \mathrm{~m}$ & 2285 v. h. \\
\hline 24 & Eiche & 3493705 & 5832365 & $+32.19 \mathrm{~m}$ & nach 2239 v. h. \\
\hline 25 & Erle & 3493680 & 5832242 & $+32.01 \mathrm{~m}$ & \\
\hline 26 & Eiche & 3493657 & 5832242 & $+32.04 \mathrm{~m}$ & nach 2141 v. h. \\
\hline 27 & Erle & 3493662 & 5832252 & $+32.06 \mathrm{~m}$ & \\
\hline 28 & Eiche & 3493648 & 5832257 & $+31.81 \mathrm{~m}$ & nach $2400 \mathrm{v} . \mathrm{l}$ \\
\hline 29 & Eiche & 3493655 & 5832260 & $+32.01 \mathrm{~m}$ & nach 2394 v. h. \\
\hline 30 & Erle & 3493665 & 5832314 & $+32.05 \mathrm{~m}$ & \\
\hline 31 & Eiche & 3493663 & 5832355 & $+31.91 \mathrm{~m}$ & nach $2147 \mathrm{v} . \mathrm{h}$. \\
\hline 32 & Erle & 3493667 & 5832393 & $+32.12 \mathrm{~m}$ & \\
\hline 33 & Eiche & 3493655 & 5832326 & $+32.05 \mathrm{~m}$ & nach 2204 v. 1 \\
\hline 34 & Erle & 3493655 & 5832307 & $+31.99 \mathrm{~m}$ & \\
\hline 35 & Eiche & 3493654 & 5832277 & $+32.21 \mathrm{~m}$ & 2138 v. h. \\
\hline 36 & Eiche & 3493645 & 5832278 & $+32.17 \mathrm{~m}$ & nach 2141 v. h. \\
\hline 37 & Erle & 3493645 & 5832225 & $+32.03 \mathrm{~m}$ & \\
\hline 38 & Erle & 3493645 & 5832220 & $+31.86 \mathrm{~m}$ & \\
\hline 39 & Eiche & 3493655 & 5832218 & $+32.13 \mathrm{~m}$ & nach 2149 v. h. \\
\hline 40 & Esche & 3493640 & 5832210 & $+32.14 \mathrm{~m}$ & \\
\hline 41 & Eiche & 3493644 & 5832215 & $+32.06 \mathrm{~m}$ & $2190 \mathrm{v.} 1$ \\
\hline 42 & Eiche & 3493630 & 5832205 & $+32.33 \mathrm{~m}$ & \\
\hline 43 & Eiche & 3493610 & 5832188 & $+32.15 \mathrm{~m}$ & nach 2142 \\
\hline
\end{tabular}

Bei der späteren Aufbereitung des Probenmaterials zeigte sich, daß von den 40 Hölzern 30 Eichen und eine Esche (Baum Nr. 40) waren, während die neun restlichen als Erlen bestimmt wurden ${ }^{2}$ ).

Das Siedener Hochmoor ist das mittlere (Sulinger Moor im W, Großes Borsteler Moor im E) von drei großen N-S verlaufenden Hochmooren, die die Ebene SE von Sulingen (Abb. 2) zwischen dem Südrand der Wildeshausener Geestplatte und dem Stauchendmoränenwall der Böhrde bedecken. Die zwischen den Mooren nach S der Großen Aue zufließenden Vorfluter Allerbeeke und Siede wurden in den 50er Jahren kanalisiert.

2) Die holzanatomische Untersuchung verdanken wir Herrn Prof. Dr. H. SACHSSE, Institut für Forstbenutzung, Göttingen. 


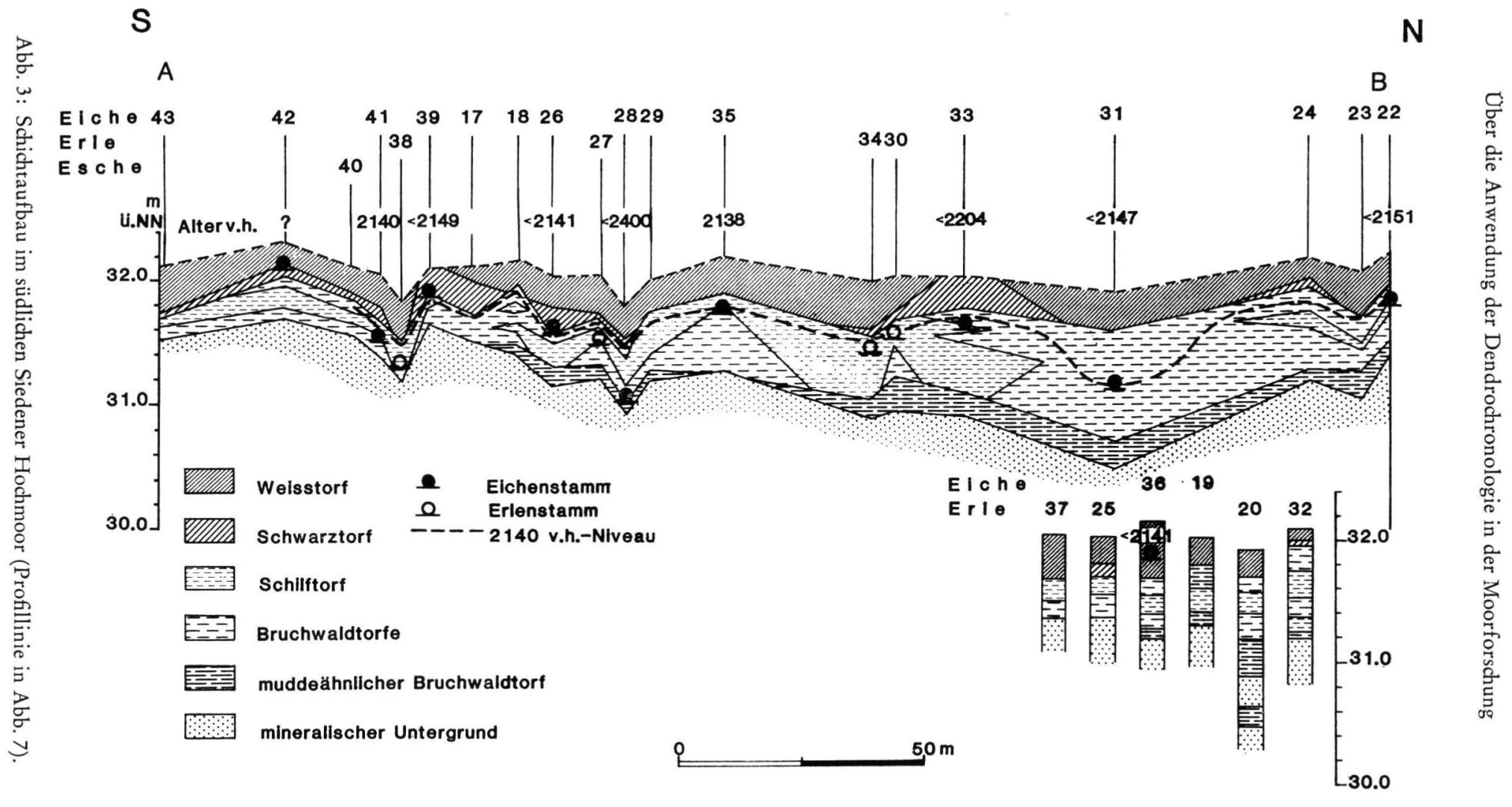


Die Moore sind auf eine Ebene aufgewachsen, die in ihrem oberen Teil während der Weichsel-Kaltzeit aus fluviatilen Sanden aufgebaut wurde. Hauptsächlich sind Fein- bis Mittelsande geschüttet worden, die an der Oberfläche unter dem Einfluß des periglazialen Klimas einer sehr starken äolischen Umlagerung ausgesetzt waren. Zahlreiche einzelne Flugsandkuppen und große zusammenhängende Flugsandfelder charakterisieren vor allem die südliche Umgebung des Siedener Moores. Vereinzelt ragen die Kuppen sogar aus dem Moor heraus. Häufig sind sie jedoch noch von Torf bedeckt und nur in den Entwässerungsgräben für die industrielle Torfgewinnung zu erkennen. Die fluviatil gebildete Ebene entspricht zeitlich der Niederterrasse. Eine Zweiteilung wie an der Ems ist nicht erfolgt, da die entsprechend großen Vorfluter fehlen.

\subsection{Einzelne Baumbefunde verschiedener Herkunft}

Als Untersuchungsmaterial standen auch Proben einzelner bei Kultivierungs- und Bauarbeiten zutage geförderter Eichen aus dem Raum Sulingen/Diepholz zur Verfügung. Lediglich auf einen dieser Stämme sei hier näher eingegangen. Für die übrigen Funde liegen weder ${ }^{14} \mathrm{C}$-Daten vor, noch ließen sie sich bislang jahrringchronologisch zu anderen Hölzern in Beziehung setzen.

Die erwähnte Eiche lag in einem Wäldchen $1 \mathrm{~km}$ nördlich der Ortschaft Barver und war offenbar bei Kultivierungsarbeiten im angrenzenden mittleren Teil des Wietingsmoores, dem Donstofer Moor, aus dem Untergrund gezogen worden (Abb. 1).

Bl. 3317 Barver der TK 25: R 3472500

H 5833400

\section{Ergebnisse}

\subsection{Entstehung und Schichtaufbau des Hochmoores bei Sieden}

Die in diesem Moor gefundenen Eichen liegen nur etwa 200-450 m von der ursprünglichen Moorgrenze im S entfernt. Die gesamte Mächtigkeit des Profils ist am Fundort nicht mehr erhalten, da vor dem Einsatz der Torfstechmaschine bereits einige dm Torf an der Mooroberfläche durch Planieren und Abbunken beseitigt worden sind. Die noch vorhandene Moorauflage hat eine Mächtigkeit zwischen 0,5 und 1,5 m. An allen hier berücksich-

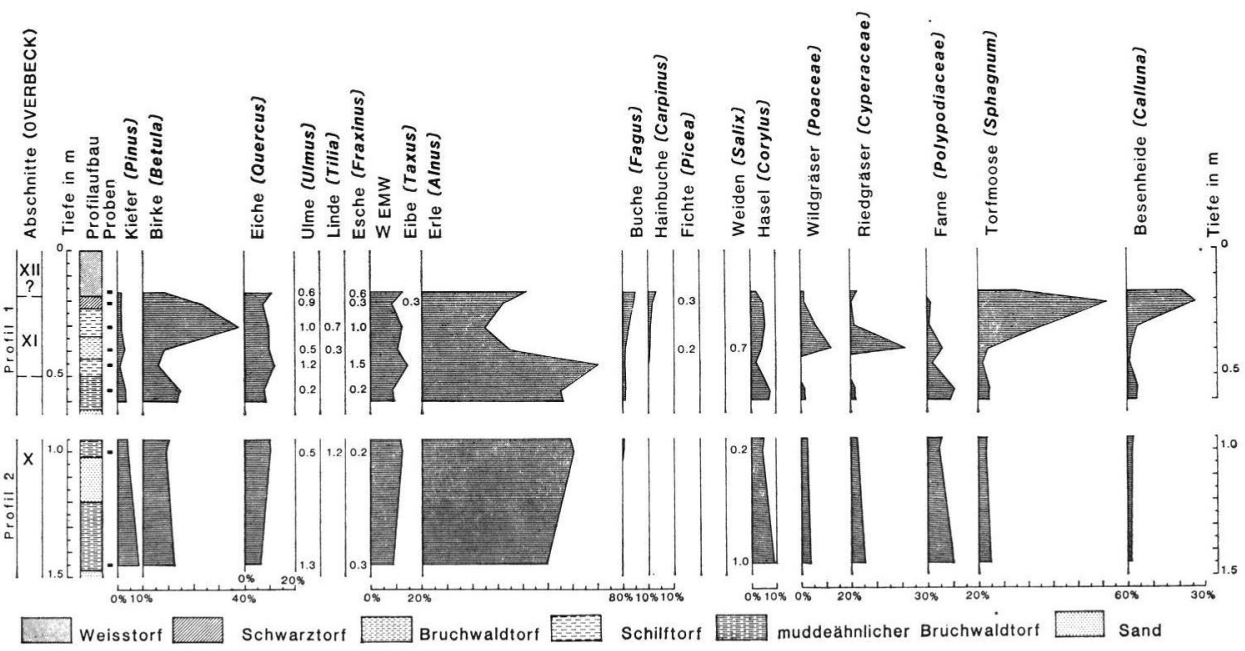

Abb. 4: Pollendiagramm aus dem Hochmoor bei Sieden (berechnet in \% der Pollensumme). 
tigten Baumfundorten ist eine Bohrung zur Beschreibung des Schichtaufbaus niedergebracht worden, die die gleiche Nummer wie der Stamm trägt. Aus 21 Bohrungen wurde ein einnivellierter Moorschnitt konstruiert, der in Abb. 3 dargestellt ist (vgl. auch Abb. 7). Weitere fünf Bohrungen, die zu weit von dieser Schnittlinie entfernt liegen, sind als Einzelbohrungen in $\mathrm{Abb} .3$ hinzugefügt.

Im Untergrund des untersuchten Moorteiles wurden auf der $250 \mathrm{~m}$ langen Strecke des Schnittes Höhendifferenzen der Pleistozänoberfläche von 1,25 m (31.72-30.47 m über NN) gemessen. Die Bohrungen 31 und 20 erfaßten eine bis $80 \mathrm{~cm}$ in den mineralischen Untergrund eingeschnittene, heute vermoorte Rinne. Weitere flachere und schmale Senken, die im Schnitt deutlich werden, mögen ebenfalls Rinnen darstellen. Das Urmeßtischblatt von 1897 zeigt zwischen den drei Hochmooren der Ebene fließende, damals z. T. noch natürlich erhaltene Bachsysteme, die an Stelle eines Flüßchens oder Baches in ein engmaschiges Netzwerk von kleinen Bächen aufgelöst sind. Die natürliche Vegetation dieser Täler waren Auenwälder auf Mineralböden.

Diese Bachnetze waren früher sicher breiter als noch 1897 und wurden durch die Moorausbreitung eingeengt. Wo im Randbereich solcher Fließsysteme die Strömungsgeschwindigkeit des Grundwassers sehr gering wird, ist ein Übergreifen von Niedermoor auf solche ehemaligen Auenbereiche möglich. Bei einer solchen Vielzahl von Fließrinnen werden immer wieder einige stillgelegt und zu Altwässern.

Die Kenntnisse über den Schichtaufbau und die Ausdeutung einer pollenanalytischen Untersuchung von zwei Profilen ${ }^{3}$ ) (Abb. 4), für die wir Herrn Dr. H. MüLler von der Bundesanstalt für Geowissenschaften und Rohstoffe, Hannover, sehr zu Dank verpflichtet sind, ermöglicht es, den Ablauf der Moorbildung zu beschreiben.

Bei hoch anstehendem, während der Wintermonate lange Zeit auch über der Oberfläche stehendem Grundwasser wuchs in der Rinne ein Erlenbruchwald. Der von ihm hinterlassene braune bis hellbraune Torf ist wegen der lang andauernden Überflutung sehr muddeähnlich und extrem stark zersetzt. Er enthält stets mehr oder weniger viele Sandpartikel, wird an einer Stelle des Tälchenquerschnittes (Bohrung 20) auch von einer fast $20 \mathrm{~cm}$ dicken Sandlinse unterbrochen, die durch kurzzeitig fließendes Wasser eingelagert wurde. Diese ältesten Rinnentorfe sind um die Mitte des letzten vorchristlichen Jahrtausends gebildet worden (ausgehender Abschnitt X nach Overbeck).

$\mathrm{Zu}$ dieser Zeit siedelte auf der grundwassernahen, aber nicht überschwemmten Umgebung des Tales ein dem heutigen Eichen-Hainbuchenwald nahestehender Eichenwald. Dieser ging sehr bald nach einem Grundwasseranstieg ebenfalls in einen Erlenbruchwald über, der einen dunkelgrauen bis dunkelgraubraunen, sonst dem der Rinnenfüllung ganz entsprechenden Torf bildete. Diese basalen, muddeähnlichen Torfe haben insgesamt eine Mächtigkeit von 5 bis $25 \mathrm{~cm}$. Der größere Teil des untersuchten Moorausschnittes war demnach winterlichen Überflutungen ausgesetzt. Nur am Südrand der Fläche, wo der Untergrund des heutigen Moores am höchsten ansteigt, blieb der nicht torfbildende Eichenwald für höchstens wenige Jahrhunderte erhalten (Bohrung 43-17, vgl. Abb. 3).

Uber die Torfunterlage des tiefer liegenden Abschnittes schob sich aus dem Zentrum des weiter nördlich wohl schon länger bestehenden Moores in kontinuierlichem Ablauf eine andere Erlenbruchwaldgesellschaft vor, in der Eichen, Birken (Betula pubescens) und einzelne Eschen (Fraxinus excelsior) wuchsen. Dieser Wald bildete während eines Zeitraumes von etwa zwei Jahrhunderten einen stark zersetzten, holzhaltigen, stellenweise schilfreichen Torf von 20 bis $60 \mathrm{~cm}$, in der Rinne auch $90 \mathrm{~cm}$ Mächtigkeit. In diesen Bruchwaldtorf ist ein seggenreicher Schilftorf eingeschaltet, der mehr oder weniger holzfrei ist. Auf dem Moor haben demnach neben bewaldeten Partien, die mit Weidengebüsch umman-

3) Das vollständige Pollendiagramm kann im Archiv des NLfB eingesehen werden. 
telt waren, offene Inseln mit übermannshohem Schilf (Phragmites australis) in engem räumlichem und zeitlichem Wechsel bestanden. Gerade in solchen Schilfdickichten kann im Winter bis zu $50 \mathrm{~cm}$ Wasser über der Oberfläche stehen (s. TüxeN 1967). Diese Überstauung ist mehr durch kaum noch abfließendes Niederschlagswasser bedingt und nicht durch von außerhalb des Moores zugeführtes Wasser entstanden wie in der vorangegangenen Primärphase der Moorentwicklung.

Im jüngsten Abschnitt des Bruchwaldtorfes, der pollenanalytisch auf die ersten ein bis zwei Jahrhunderte v. Chr. Geburt datiert wird (früher Abschnitt XI), zeigen sich deutliche Veränderungen. Der Erlenanteil der torfbildenden Waldvegetation geht zugunsten der Birke stark zurück, im Unterwuchs treten Kräuter und hohe Stauden zurück und machen Moosen, ja selbst Torfmoosen (vermutlich Arten wie Sphagnum fimbriatum und S. palustre) und wohl auch schon Wollgräsern Platz. Der ursprünglich nährstoffreiche Erlenbruchwald ist zu einem erheblich nährstoffärmeren und saureren Birkenbruchwald geworden, wofür auch ein Nachweis der Hochmoorschlenkenart Scheuchzeria palustris spricht. Im Bereich der Bohrungen 29 bis 30 ist während dieser Zeit ein ebenso verarmter Schilftorf entstanden. In diesem oberen Abschluß des Niedermoores liegt die Mehrzahl der Eichenstämme, alle Erlen und auch die einzelne Esche.

Dieser deutliche Wechsel in Vegetation und Torfbildung ist eine Folge des verlangsamten und schließlich zum Stillstand kommenden Grundwasseranstieges. Die zunehmende und in Zukunft ausschließliche Versorgung mit Niederschlagswasser läßt die Vegetation anscheinend kurzfristig in ein von lebenden Bäumen freies, von Torfmoosen der Acutifolia-Sektion beherrschtes Hochmoor umschlagen. Nur an einer Stelle (Bohrung 33) beginnt die Hochmoorbildung mit einer sehr nassen Phase, einer Schlenke mit Sphagna sect. Cuspidata, während überall sonst trockenere Stadien mit Besenheide (Calluna vulgaris) und Scheiden-Wollgras (Eriophorum vaginatum) direkt über dem Birkenbruchwald bzw. dem Schilftorf auftreten. Der zunächst gebildete Hochmoortorf ist in der Regel ein stark zersetzter sog. Schwarztorf, der meist nur Mächtigkeiten bis höchstens $20 \mathrm{~cm}$ erreicht. Darüber folgt, z. T. auch unmittelbar auf dem liegenden Niedermoortorf, schwach zersetzter Hochmoortorf (Weißtorf), der heute abgebaut wird. Zwei Eichenstämme liegen im Hochmoortorf, Baum 42 im Schwarztorf, Baum 36 im Weißtorf.

\subsection{Dendrochronologischer Befund}

\subsubsection{Methodis ches}

Um die Möglichkeiten und Grenzen der Jahrringchronologie verständlich zu machen, seien zunächst einige methodische Besonderheiten herausgestellt. Hinsichtlich weiterer Einzelheiten sei auf Huber (1969) und Delorme (1973, 1979) hingewiesen.

Die dendrochronologische Datierung einer Holzprobe gelingt nur dann, wenn für die entsprechende Baumart und regionale Herkunft eine Vergleichschronologie zur Verfügung steht und beide Kurven sich um mindestens etwa 100 Jahre überlappen. Mit zunehmender Überlappungslänge steigen die Aussichten auf eine erfolgreiche Datierung beträchtlich. Sie verbessern sich auch dann, wenn zunächst mehrere Holzproben desselben Fundortes untereinander relativ datiert werden können und somit eine mehrfach belegte Mittelkurve für den Synchronisationsversuch mit der Standardchronologie zur Verfügung steht.

Da eine Jahrringkurve mit einer anderen entweder auf das Jahr genau oder gar nicht in zeitliche - relative oder absolute - Ubereinstimmung gebracht werden kann, kennt die Dendrochronologie in diesem Sinne keine Fehlergrenzen. Solche können aber sehr wohl bei der Ableitung des Absterbejahres des Ursprungsbaumes auftreten. Dieses läßt sich nur dann exakt festlegen, wenn die Holzprobe noch die „Waldkante“ aufweist, d. h. den letzten vor dem Absterben angelegten Zuwachsring. Ist dieses Merkmal nicht gegeben, 
entsteht ein Datierungsspielraum durch die ungewisse Anzahl fehlender Jahrringe. Bei der Kernholzart Eiche erlauben erhaltene Reste des äußeren andersfarbigen und leider weniger dauerhaften Splintholzes die Bestimmung des Absterbejahres innerhalb eines Rahmens von maximal \pm 6 Jahren. Dies ist möglich, weil die mittlere Anzahl der Splintringe und ihre Streuung bekannt sind. Fehlt ein Splintrest, so bleibt ungewiß, wieviel Kernholzringe neben dem Splint verlorengegangen sind. Hier kann dann i. a. nur ein terminus post, ein frühest mögliches Absterbejahr, angegeben werden, indem der Datierung des Endjahres der Ringfolge die Minimalzahl von Splintringen hinzugezählt wird. Wieviele Jahre später der Baum u. U. tatsächlich abgestorben ist, muß in diesem Fall offenbleiben.

Aus dem für diese Arbeit zur Verfügung stehenden Material wurden die ringärmsten Hölzer mit weniger als 90 Jahrringen ausgesondert. Danach verblieben 29 Eichen aus dem Borsumer Moor mit bis zu 294 Jahrringen, 30 Eichen aus dem Siedener Hochmoor mit bis zu 267 Ringen sowie einige Einzelfunde verschiedener Herkunft mit maximal 331 Jahrringen.

Bei den Borsumer Hölzern ist leider in keinem Fall ein Splintrest erhalten, während unter den Siedener Proben immerhin vier mit Waldkante und weitere drei mit Splintresten sind. Auch die Eiche aus dem Donstorfer Moor weist noch Waldkante auf.

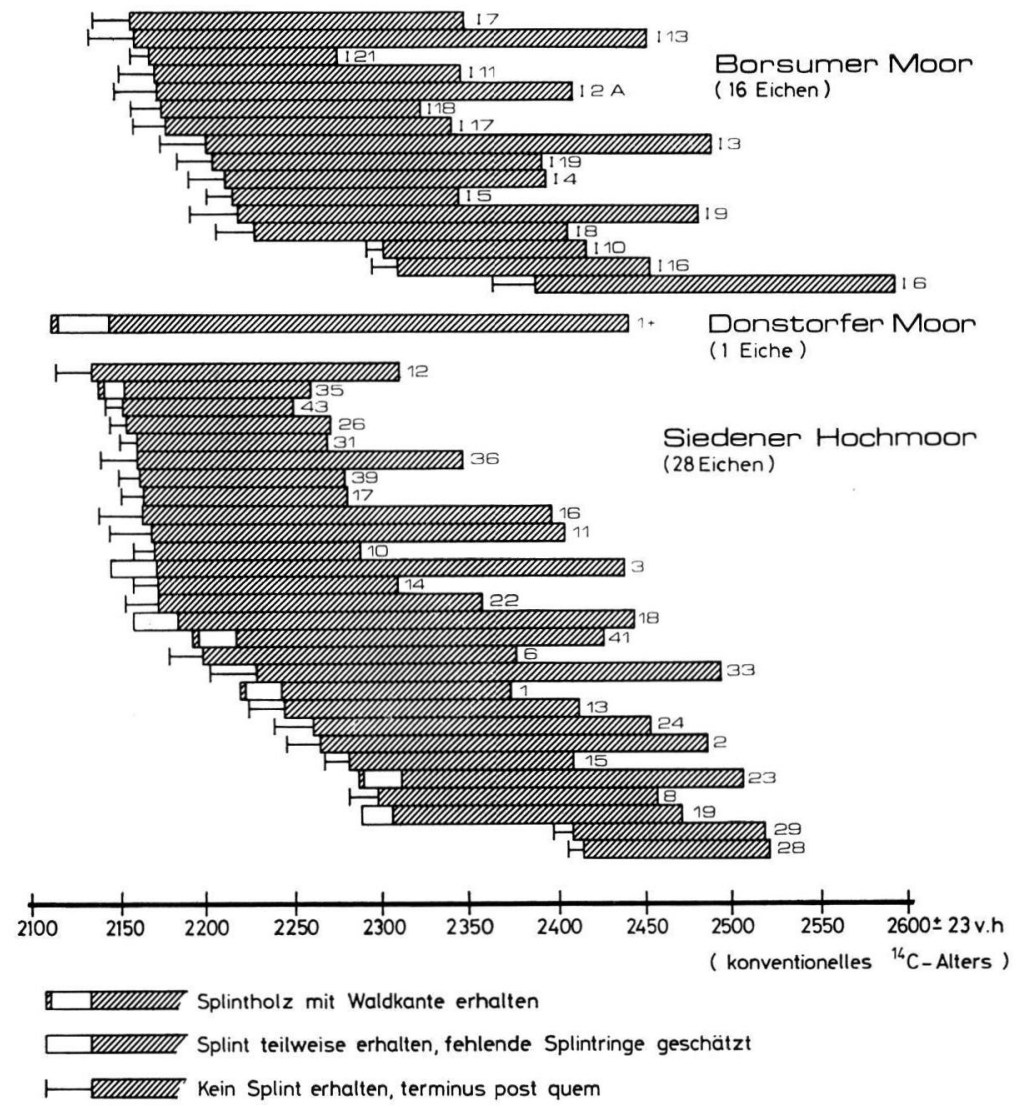

Abb. 5: Synchronisierte Eichen aus den untersuchten Mooren. 


\subsubsection{A ufbau von Standortkurven}

Da regionale Eichenchronologien für die Fundorte nicht zur Verfügung standen, beschränkten sich die Synchronisationsversuche zunächst auf den Vergleich aller Einzelkurven eines Standortes in allen untereinander möglichen Kombinationen. Sobald sich synchrone Kurvenpaarungen ergaben, wurden die überbrückten Einzelkurven zu Mittelkurven vereinigt und diese an ihrer Stelle für weitere Synchronisationsversuche benutzt. Danach stellte sich folgendes Zwischenergebnis ein:

\section{Borsumer Moor}

Fundstelle 1: Mittelkurve Borsum 1 mit 437 Jahren aus 16 synchronen Eichen. Mittelkurve Borsum 2 mit 214 Jahren aus 3 synchronen Eichen. Zwei Hölzer lassen sich nicht synchronisieren.

Fundstelle 2: Mittelkurve Borsum 4 mit 225 Jahren aus 3 synchronen Eichen. Mittelkurve Borsum 6 mit 226 Jahren aus 2 synchronen Eichen. Drei Hölzer lassen sich nicht synchronisieren.

$\mathrm{S}$ iedener $\mathrm{Hoch}$ moor

Mittelkurve Voigtei 1 mit 383 Jahren aus 28 synchronen Eichen. Zwei Proben lassen sich nicht synchronisieren.

Donstorfer $\mathrm{M}$ oor

Von der einzelnen Eiche mit 331 Jahrringen wurden die sechs äußersten, extrem schmalen Ringe nicht gemessen. Für weitere Kurvenvergleiche stand daher eine Ringfolge von 325 Jahren zur Verfügung.

Der Vergleich der Mittelkurven untereinander führte dann zu dem überraschenden Ergebnis, daß sich die beiden längsten und am häufigsten belegten, nämlich Borsum 1 und Voigtei 1, als miteinander und auch zu der Einzelprobe aus dem Donstorfer Moor syn-

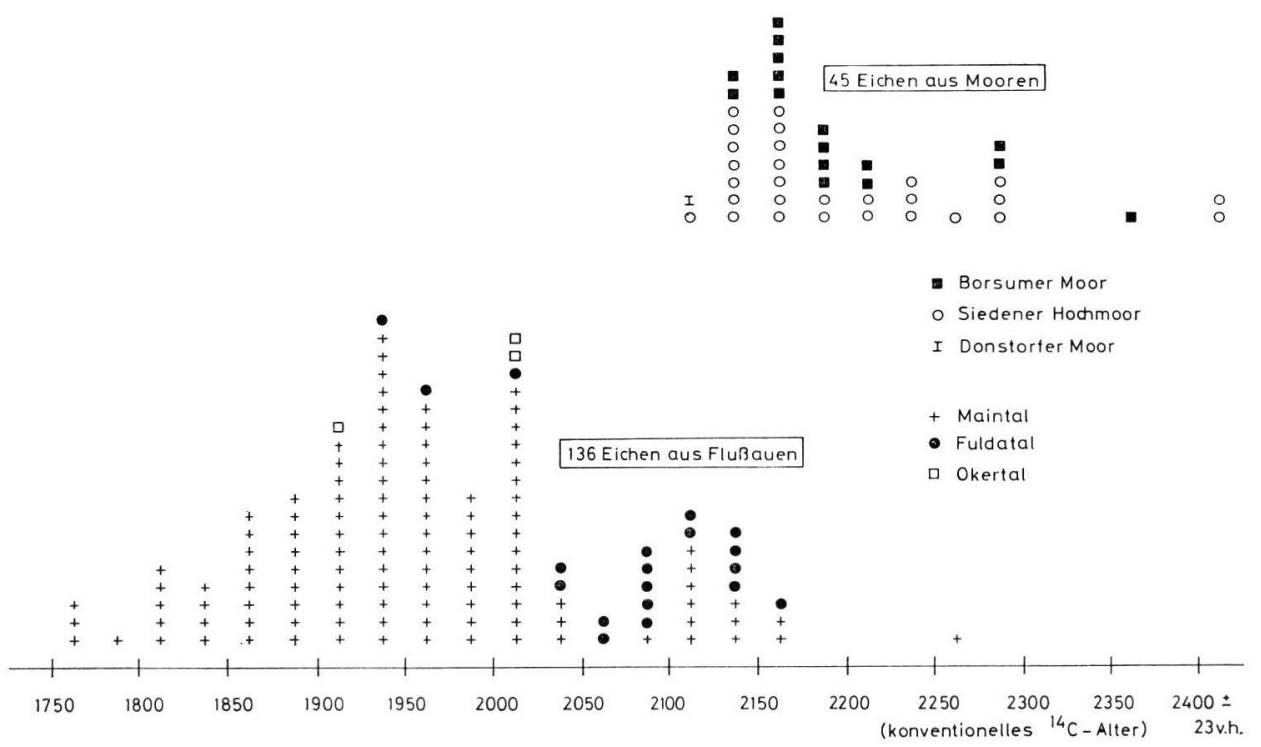

Abb. 6: Häufigkeitsverteilung der Absterbejahre subfossiler Eichen aus Flußtälern und Mooren. 
chron erwiesen. Die somit insgesamt 45 überbrückten Eichen bilden die Teilchronologie Moor 1 mit 474 Jahren. Die von diesen Bäumen belegten Zeiträume und die Aufeinanderfolge ihrer Absterbedaten sind in Abb. 5 dargestellt.

\subsubsection{Synchronisierung der Eichen aus Mooren mit der eisenzeitlichen Auwaldeichenchronologie}

Aufgrund eines mittlerweile vorliegenden ${ }^{14} \mathrm{C}$-Datums (Hv 10.246, s. Abschnitt 3.3) war es möglich, den von Moor 1 belegten Zeitraum auch absolut mit vorläufig etwa 2100 bis 2600 v. h. anzugeben. Damit war eine zeitliche Überlappung mit der in Göttingen aus subfossilen Auwaldeichen von Main, Fulda, Werra und Oker aufgebauten eisenzeitlichen Chronologie wahrscheinlich. Tatsächlich gelang es, beide Chronologien zu synchronisieren, wodurch

- die Auwaldeichenchronologie um 215 Jahre zum Alteren auf insgesamt 811 Jahre verlängert wurde,

- der genaue Zeitabstand zwischen dem Absterben der Auwaldeichen einerseits und der Eichen aus Mooren andererseits abzuleiten ist,

- die für die Auwaldeichenchronologie vorliegenden sieben ${ }^{14} \mathrm{C}-\mathrm{Daten}$ mit dem ${ }^{14} \mathrm{C}-\mathrm{Da}$ tum für Moor $1 \mathrm{zu}$ einem Wert mit vergleichsweise geringer Fehlerstreuung gemittelt werden können.

Die Synchronisation der Mittelkurve Moor 1 mit der eisenzeitlichen Auwaldeichenchronologie legt die Frage nahe, ob möglicherweise die Einlagerung von Eichen im Moor wie im Flußschotter auf die gleiche - klimatische - Ursache zurückgeführt werden kann. Näheren Aufschluß über den zeitlichen Zusammenhang beider Entwicklungen vermittelt eine Häufigkeitsverteilung der Absterbedaten (Abb. 6). Dabei wird deutlich, daß das Absterben der Eichen aus Mooren im wesentlichen zwischen 2300 und 2100 v. h. mit Höhepunkt um 2150 v. h. erfolgte, während die Eichen im Maintal in der Zeit von 2160 bis 1750 v. h. abstarben, mit Kulmination zwischen 2025 und 1925 v. h., also erst 125 bis 225 Jahre später. Im Fuldatal scheint die Auwaldzerstörung etwa 150 Jahre früher als am Main ihren Höhepunkt erreicht zu haben. Für eine eindeutige Feststellung in dieser Richtung ist jedoch die Anzahl der Eichen aus dem Fuldatal noch zu gering.

\subsubsection{A bsterbedaten}

Aus Abb. 5 lassen sich mehrere wichtige Erkenntnisse gewinnen. So hat sich das Absterben der Eichen über einen Zeitraum von etwa 300 Jahren hingezogen, wobei diese Entwicklung im Borsumer Moor wie im Siedener Hochmoor in auffälliger Parallelität in die Zeit von 2400 bis 2100 v. h. fiel. Selbst wenn man berücksichtigt, daß die Absterbejahre bei den meisten Proben nur als termini post angegeben werden können, belegen doch allein schon die wenigen Bäume mit Waldkante oder Splintresten den größten Teil des angegebenen Zeitraumes sicher.

$\mathrm{Zu}$ einer eingehenderen Analyse der Absterbedaten muß der Lageplan der Siedener Holzfunde (Abb. 7) mit herangezogen werden. Die Absterbedaten häufen sich zwischen 2160 und 2135 v.h. Bäume aus dieser Zeit kommen über die ganze Aufnahmefläche verteilt vor.

Auffallend sind die vergleichsweise großen Zeitdifferenzen zwischen nahe beieinander liegenden Bäumen.

Der jahrringchronologische Befund erlaubt auch Rückschlüsse auf die Keimjahre verschiedener Eichen. Ist die ausgewertete Baumscheibe nahe dem Stammfuß entnommen und 


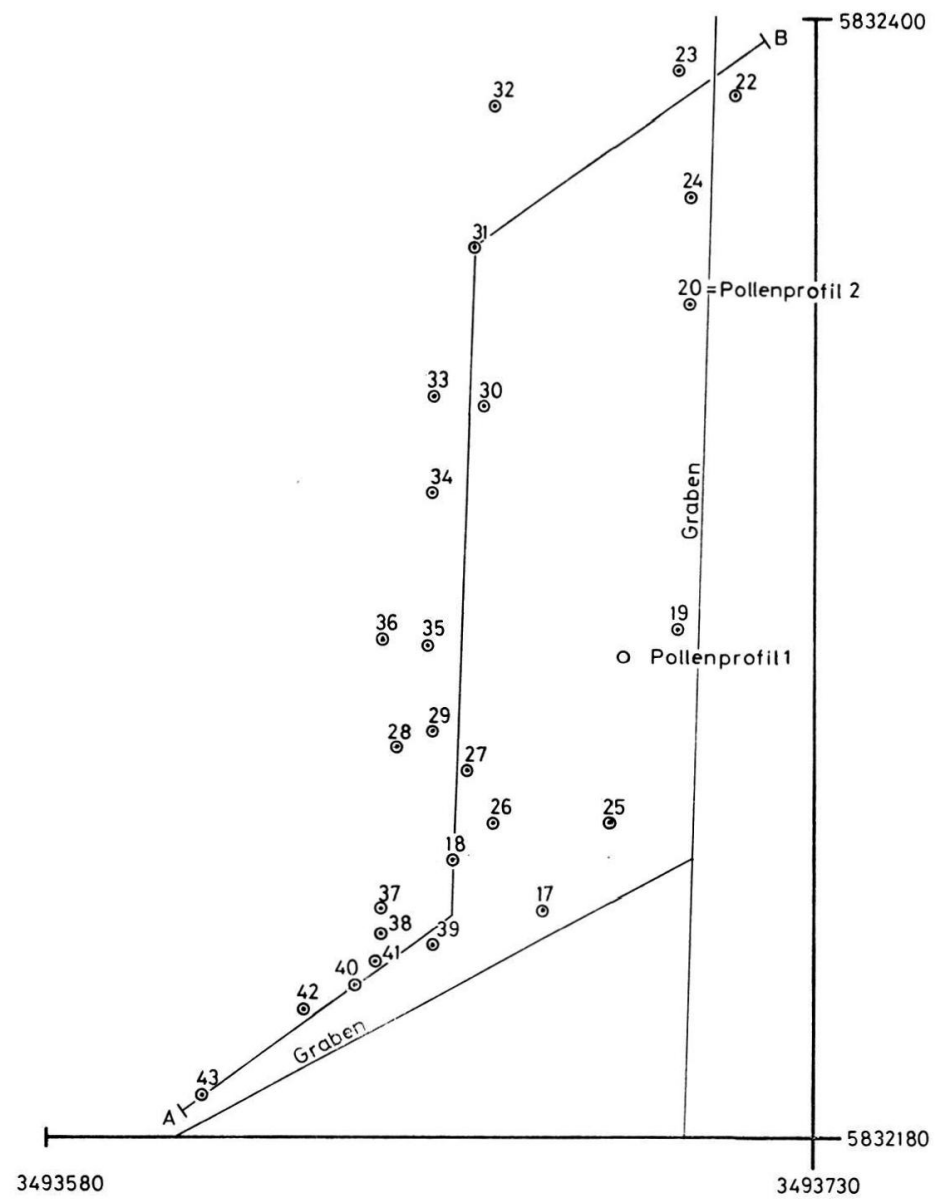

Abb. 7: Lage der Bäume 17-43, der Pollenprofile und der Profillinie im Hochmoor bei Sieden (vgl. Abb. 3).

bis zum innersten, ältesten Holz, dem Mark, erhalten, dann kann die Datierung des ältesten Jahrringes nur um wenige Jahre jünger als das Keimjahr ausfallen.

Auf diese Weise läßt sich für die Eiche Nr. 35 nachweisen, daß sie zwischen 2260 und 2270 v. h. gekeimt ist, zu einer Zeit also, als z. B. die Bäume Nr. 19 und 23 bereits mindestens 15 Jahre abgestorben waren.

Leider kann die Dendrochronologie keinen Aufschluß über die zeitliche Einordnung der im Siedener Hochmoor gefundenen Erlen und der einen Esche geben. Schon die Ringarmut dieses Materials (75 bis 134 Jahrringe der Erlen, 76 Jahrringe der Esche) macht Synchronisationsversuche wenig aussichtsreich. Zudem lassen sich Erlen und Eschen i.a. nicht mit einer Eichenchronologie datieren. Auch der Vergleich der Erlen untereinander blieb ohne Ergebnis. Das schließt jedoch nicht aus, daß sie möglicherweise zeitgleich sind. Wie EluING (1966) gezeigt hat, sind gerade bei Erlen Jahrringanomalien häufig, die ihre dendrochronologische Auswertung sehr erschweren. 


\subsubsection{Ahnlichkeitsbeziehungen zwischen verschiedenen Eichenjahrringkurven}

Der regionale Geltungsbereich von Jahrringchronologien wird durch die i. a. mit zunehmender Entfernung der Probenherkünfte abnehmende Übereinstimmung von Jahrringkurven begrenzt. Um zu einer Aussage zu kommen, inwieweit großräumige Klimaeinflüsse auf das Baumwachstum durch standörtlich wirksame Wachstumsfaktoren überlagert worden sind, sollen die zwischen den Eichen aus verschiedenen norddeutschen Mooren und Eichen aus süddeutschen Flußtälern bestehenden Ähnlichkeitsbeziehungen untersucht werden.

Als Maß der Übereinstimmung eines Jahrringkurvenpaares wird i. a. das Gleichläufigkeitsprozent (GL-0\%) angegeben. Es zeigt an, in wieviel Prozent der Fälle die Ausschläge der beiden Kurven in den zur Deckung gebrachten Jahresintervallen den gleichen Richtungssinn haben, ob sich also die Ringbreite in beiden Kurven gegenüber dem Vorjahr übereinstimmend vergrößert oder verkleinert hat. Je häufiger das der Fall ist, desto größer wird das GL-0\% und desto ähnlicher ist sich das Kurvenpaar. Asynchrone Kurvenkombinationen weisen im Mittel 50\% GL auf. Dabei streuen die Werte um dieses Mittel um so stärker, je kürzer der Zeitraum der Kurvenüberlappung ist. Die über $50 \%$ liegende Hälfte dieses Streubereiches überschneidet sich teilweise mit dem Streubereich der GL-Werte synchroner Lagen. Es entsteht damit das Problem, Deckungslagen mit rein zufällig hohen GL-Werten als solche zu erkennen und von der einzig möglichen synchronen Lage zu unterscheiden. Die Streufelder der GL-Werte für synchrone und Zufallslagen überschneiden sich mit zunehmender Kurvenüberlappung immer weniger, bis sie sich schließlich sogar klar voneinander trennen. Hieraus wird die große Bedeutung der Jahrringanzahl einer Holzprobe für ihre Datierbarkeit ersichtlich (HuBER 1943, EcksteIN 1969, Delorme 1973).

Zur Charakterisierung des hier bearbeiteten Materials seien im folgenden die GLWerte angegeben, die sich bei den verschiedenen s y $\mathrm{c}$ h ron en Kurvenkombinationen ergaben. Dabei können als ungefährer Anhalt für das Maß der Übereinstimmung bei einer Kurvenüberlappung von mindestens 150 Jahren folgende Rahmenwerte gelten:

$$
\begin{aligned}
& 73-80 \%=\text { ausgezeichnete Übereinstimmung } \\
& 68-72 \%=\text { sehr gute Übereinstimmung } \\
& 65-67 \%=\text { Gute Übereinstimmung } \\
& 62-64 \%=\text { Mäßige Übereinstimmung } \\
& 59-61 \%=\text { kaum noch erkennbare Úbereinstimmung. }
\end{aligned}
$$

Werte über $80 \%$ GL kommen kaum vor, solche unter $59 \%$ lassen i. a. keine Ǔbereinstimmung mehr erkennen.

Die Homogenität eines Probenkollektivs kann durch das GL-0/o gekennzeichnet werden, das die Einzelkurven untereinander in allen möglichen synchronen Paarungen durchschnittlich auf weisen.

Tabelle 2 enthält die entsprechenden Werte für das Moormaterial und die eisenzeitlichen Eichen aus dem Main- und Fuldatal.

Tabelle 2: Mittlere Gleichläufigkeit von Eichenhölzern bestimmter Herkunft

\begin{tabular}{crcc}
\hline \multicolumn{2}{c}{ Eichen aus Mooren } & \multicolumn{2}{c}{ Eichen aus Flußtälern } \\
\hline Borsum & Sieden & Maintal & Fuldatal \\
$61,5 \%$ & $62,2 \%$ & $64,4 \%$ & $63,0 \%$
\end{tabular}


Es wird deutlich, daß die Eichen aus Flußtälern insgesamt homogener gewachsen sind als die Eichen aus Mooren. Dabei liegen im Maintal die Probenherkünfte bis zu $60 \mathrm{~km}$, im Fuldatal bis zu $12 \mathrm{~km}$ auseinander, während die Eichen aus Mooren jeweils innerhalb eines Radius von nur wenigen hundert Metern geborgen wurden. Hier müssen kleinstandörtliche Unterschiede das Baumwachstum deutlich stärker beeinflußt haben als in den Auwäldern an Main und Fulda.

Der Vergleich der verschiedenen Standort- und Regionalchronologien (Tab. 3) soll die Ahnlichkeitsbeziehungen zwischen ihnen aufzeigen:

Tabelle 3: Gleichläufigkeitswerte und Uberlappungslängen synchroner Regional- und Standortmittelkurven

\begin{tabular}{|c|c|c|c|c|c|}
\hline & Moor 1 & Borsum 1 & Vogtei 1 & $\begin{array}{l}\text { Auwald } \\
\text { Fulda }\end{array}$ & $\begin{array}{l}\text { Auwald } \\
\text { Main }\end{array}$ \\
\hline Moor 1 & - & - & - & $\begin{array}{c}62,4 \% \\
189 \text { Jahre }\end{array}$ & $\begin{array}{c}64,7 \% \\
268 \text { Jahre }\end{array}$ \\
\hline Borsum 1 & - & - & $\begin{array}{c}64,7 \% \\
360 \text { Jahre }\end{array}$ & $\begin{array}{c}56,9 \% \\
152 \text { Jahre }\end{array}$ & $\begin{array}{c}55,4 \% \\
231 \text { Jahre }\end{array}$ \\
\hline Vogtei 1 & - & $\begin{array}{c}64,7 \% \\
360 \text { Jahre }\end{array}$ & - & $\begin{array}{c}62,1 \% \\
\text { 174 Jahre }\end{array}$ & $\begin{array}{c}64,4 \% \\
253 \text { Jahre }\end{array}$ \\
\hline $\begin{array}{l}\text { Auwald } \\
\text { Fulda }\end{array}$ & $\begin{array}{c}62,4 \% \\
189 \text { Jahre }\end{array}$ & $\begin{array}{c}56,9 \% \\
152 \text { Jahre }\end{array}$ & $\begin{array}{c}62,1 \% \\
\text { 174 Jahre }\end{array}$ & - & $\begin{array}{c}72,7 \% \\
343 \text { Jahre }\end{array}$ \\
\hline $\begin{array}{l}\text { Auwald } \\
\text { Main }\end{array}$ & $\begin{array}{c}64,7 \% \\
\text { 268 Jahre }\end{array}$ & $\begin{array}{c}55,4 \% \\
231 \text { Jahre }\end{array}$ & $\begin{array}{c}64,4 \% \\
253 \text { Jahre }\end{array}$ & $\begin{array}{c}72,7 \% \\
343 \text { Jahre }\end{array}$ & - \\
\hline
\end{tabular}

Es fällt besonders auf, daß die beiden Moorherkünfte untereinander mit 64,7 \% $\mathrm{kei}$ nen besseren Wert aufweisen als das Gesamtmoormaterial (Moor) zur Mainchronologie. Die beiden Auwaldchronologien zeigen dagegen untereinander mit 72,7 \% GL einen deutlich höheren Wert. Durch die folgende Tabelle läßt sich diese Aussage noch ergänzen.

Tabelle 4: Mittlere Gleichläufigkeitswerte beim Vergleich von Einzelkurven verschiedener Herkunft mit Regional- und Standortmittelkurven

\begin{tabular}{|c|c|c|c|c|c|c|}
\hline & \multicolumn{5}{|c|}{$\mathrm{Mit}$ e $1 \mathrm{k}$ u r ve $\mathrm{n}$} \\
\hline & & Moor 1 & Borsum 1 & Vogtei 1 & $\begin{array}{l}\text { Auwald } \\
\text { Fulda }\end{array}$ & $\begin{array}{c}\text { Auwald } \\
\text { Main }\end{array}$ \\
\hline \multirow{5}{*}{ 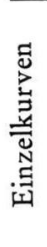 } & Borsumer Moor & $66,5 \%$ & $72,1 \%$ & $60,5 \%$ & $53,9 \%$ & $54,5 \%$ \\
\hline & Siedener Moor & $71,1 \%$ & $60,6 \%$ & $72,4 \%$ & $54,1 \%$ & $57,1 \%$ \\
\hline & Donstorfer Moor & $68,2 \%$ & $62,0 \%$ & $62,8 \%$ & $60,1 \%$ & $61,6 \%$ \\
\hline & Fuldatal & $59,3 \%$ & $54,1 \%$ & $60,1 \%$ & $73,9 \%$ & $65,6 \%$ \\
\hline & Maintal & $55,3 \%$ & $54,6 \%$ & $58,6 \%$ & $65,4 \%$ & $73,7 \%$ \\
\hline
\end{tabular}

Während die Auwaldchronologien für Main und Fulda zu den Einzelkurven der jeweils anderen Auwaldeichenherkunft mittlere GL-Werte von gut $65 \%$ aufweisen und damit eine i. a. für eine Datierung ausreichende Übereinstimmung zeigen, erreichen die Moormittelkurven zu den Einzelkurven der jeweils anderen Moorherkunft mittlere GL-Werte von nur gut $60 \%$, die eine für eine Synchronisierung i. a. nicht mehr ausreichende Kurvenübereinstimmung anzeigen. 
Danach erscheint es erforderlich, zur Datierung von Eichen aus norddeutschen Mooren zunächst Standortmittelkurven aufzubauen. Durch Synchronisation einer ausreichenden Zahl von Einzelbelegen werden dann aber kleinstandörtlich bedingte Kurvenausschläge soweit „herausgemittelt", daß norddeutsche Moormittelkurven sogar mit süddeutschen Auwaldeichenchronologien überbrüickt werden können. Auch die norddeutschen Eichen zeigen also im Vergleich mit süddeutschem Material letztlich noch eine so deutliche großklimatisch bedingte - Übereinstimmung des Jahrringwachstums, daß auch sie zum Aufbau einer postglazialen Eichenchronologie für Mitteleuropa herangezogen werden können.

\subsubsection{Durchschnittliche Jahrringbreite verschiedener Eichenherkünfte}

Bislang haben wir uns mit Schwankungen der Jahrringbreite als Grundlage für dendrochronologische Überbrückungen beschäftigt, wobei die absolute Ringbreite außer Betracht blieb. Aber auch diese, und zwar als durchschnittliche Jahrringbreite, verschiedener Probenkollektive, kann u. U. zu interessanten Schlüssen führen. Folgende durchschnittliche Ringbreiten wurden für das Untersuchungsmaterial ermittelt:

Tabelle 5: Durchschnittliche Ringbreiten subfossiler Eichen aus Mooren und Flußtälern

\begin{tabular}{cccc}
\hline \multicolumn{2}{c}{ Eichen aus Mooren } & \multicolumn{2}{c}{ Eichen aus Flußtälern } \\
\hline Borsumer Moor & Siedener Moor & Fuldatal & Maintal \\
$0,99 \mathrm{~mm}$ & $1,04 \mathrm{~mm}$ & $1,92 \mathrm{~mm}$ & $1,77 \mathrm{~mm}$
\end{tabular}

Während die Auwaldeichen im Mittel nahezu doppelt so breite Ringe angelegt haben wie die Eichen aus Mooren, weisen die verschiedenen Herkünfte beider Gruppen untereinander kaum nennenswerte Unterschiede auf. Der Vorsprung der Auwaldeichen bleibt im gleichen Ausmaß erhalten, wenn man nur die Ringbreiten des zeitlichen Überlappungsbereiches beider Gruppen berücksichtigt. Insgesamt haben also in den süddeutschen Flußauen für die Eichen wesentlich günstigere Wachstumsbedingungen als auf den norddeutschen Moorstandorten bestanden.

\subsection{Radiokarbondatierungen}

Dendrochronologie und ${ }^{14} \mathrm{C}$-Verfahren ergänzen sich bei der Zeitbestimmung subfossiler Hölzer ausgezeichnet, vorausgesetzt, die Auswahl der radiometrisch zu datierenden Proben aus einem zur Verfügung stehenden Kollektiv von Hölzern erfolgt nicht wahllos, sondern nach vorausgehender jahrringanalytischer Bearbeitung gezielt zur Datierung der dann ausgeschiedenen Gruppen jeweils synchroner Hölzer. Genaue relativchronologische Einordnung verschiedener Proben durch die Dendrochronologie und ihre ungefähre absolute Datierung mit Hilfe des ${ }^{14} \mathrm{C}$-Verfahrens lassen sich so zur Beantwortung unterschiedlicher wissenschaftlicher Fragen kombinieren.

Für die vier Mittelkurven aus dem Borsumer Moor teilte $\mathrm{GEYH}^{4}$ ) folgende ${ }^{14} \mathrm{C}-\mathrm{Daten}$ mit:

Hv $10.246=2130 \pm 55$ v. h., datiert Borsum 12124 bis 2560 v. h.

$\mathrm{Hv} 10.476=2340 \pm 50$ v. h., datiert Borsum 22335 bis 2548 v. h.

Hv $10.478=1925 \pm 55$ v. h., datiert Borsum 41894 bis 2118 v. h.

$\mathrm{Hv} 10.477=1985 \pm 60$ v. h., datiert Borsum 61972 bis 2204 v. h.

4) Für die 14C-Datierungen sei Herrn Dr. M. A. GEYH/NLfB herzlich gedankt. 
Es ist auffallend, daß alle ${ }^{14} \mathrm{C}$-Daten in die Zeit um Chr. Geb. bis etwa 390 davor fallen, also vergleichsweise nahe beieinander liegen. Eine jahrringchronologische Überbrükkung des so ${ }^{14} \mathrm{C}$-vordatierten Materials ist gleichwohl nicht gelungen. Dies ist nicht allein durch die dem Radiokarbonverfahren innewohnenden Ungenauigkeiten zu erklären. Diese machen durchaus eine breitere zeitliche Streuung des Materials möglich, als in den ${ }^{14} \mathrm{C}$ Daten zum Ausdruck kommt. Die wirkliche zeitliche Uberlappung der verschiedenen Jahrringfolgen wäre dann bei bestimmten Paarungen u. U. für eine jahrringchronologische Úberbrückung von vornherein zu kurz. Es ist aber auszuschließen, daß dies für alle sechs möglichen Kurvenpaarungen gilt. Es ist daher anzunehmen, daß tatsächlich mehrere synchrone Kombinationen vorliegen, die aber nicht als solche erkannt werden können, weil die synchronen Jahrringbreitenfolgen zu geringe Übereinstimmungen aufweisen. Bei den dicht benachbarten Herkünften der Eichenhölzer können dafür nicht klimatische Ursachen, sondern nur standörtlich wirksame Faktoren verantwortlich gemacht werden, die den Klimaeinfluß auf das Baumwachstum überlagert haben.

Infolge der jahrringchronologischen Überbrückung der Mittelkurven von Borsum und Voigtei mit der eisenzeitlichen Auwaldeichenchronologie konnten auch die für letzteren vorliegenden sieben ${ }^{14} \mathrm{C}$-Daten für eine verfeinerte absolute Zeitbestimmung des Moormaterials herangezogen werden. Radiokarbondaten, deren genaue relative Abstände dendrochronologisch bestimmt sind, können auf ein Fixjahr umgerechnet und gemittelt werden (GEYH 1971). Dabei verringert sich die statistisch bedingte Fehlerstreuung beträchtlich. Nach insgesamt acht Radiokarbondaten umfaßt die Kurvenkombination eisenzeitliche Auwaldeichenchronologie - Borsum - Voigtei - Einzelfund Donstorfer Moor mit einer Länge von 811 Jahren die Zeitspanne 1784 bis 2594 v. h., mit einer statistisch bedingten Streuung von \pm 23 Jahren.

Dabei belegt die Auwaldeichenchronologie die Zeit von 1784 bis 2379 v. h., die Mittelkurve Borsum die Zeit von 2158 bis 2594 v. h., die Mittelkurve Voigtei die Zeit von 2136 bis 2516 v. h. und der Einzelfund Donstorfer Moor die Zeit von 2121 bis 2445 v. h.

\section{Diskussion}

\subsection{Bildungsgeschichte des Hochmoores bei Sieden}

Aus der Kombination der im Hochmoor bei Sieden angewandten moorstratigraphischen, pollenanalytischen und dendrochronologischen Teilergebnisse lassen sich einige Schlüsse ziehen, die zu weiteren Aussagen über die Bildungsgeschichte des südlichen Teiles des Siedener Hochmoores führen.

\subsubsection{Absterben und Einlagerung der Stämme im Moor}

Selbst die ältesten Eichen mit einem Lebensalter von gut 300 Jahren hatten bei ihrem Tod die natürliche Altersgrenze noch längst nicht erreicht. Es kann im Einzelfall aber nicht sicher unterschieden werden, ob ein Baum im Zuge eines natürlichen Ausleseprozesses durch Konkurrenzdruck seiner Nachbarn abgestorben ist, oder ob dafür ein bestimmtes Stadium der Moorentwicklung verantwortlich zu machen ist. Wahrscheinlich sind die meisten Eichen und Erlen sowie die einzelne Esche, die im oberen Teil des Niedermoortorfes liegen, infolge der recht unvermittelt einsetzenden Verringerung des Nährstoffangebotes kurz vor Beginn der Hochmoorbildung abgestorben. Dafür spricht schon die Häufung der Absterbedaten in einem bestimmten Zeitraum, unabhängig vom Lebensalter der betroffenen Bäume.

Alle von uns bearbeiteten Stämme liegen mehr oder weniger waagerecht im Moor und sind von ihren Stubben, die nur selten sichtbar waren, abgebrochen. Auch der Befund, 
daß nur wenige Stämme mit Splintresten erhalten sind, spricht dafür, daß die Bäume erst eine Reihe von Jahren nach ihrem Absterben und schon ohne Krone abgebrochen und gefallen sind (vgl. auch HAYEN 1960). Die Stämme liegen in alle Himmelsrichtungen weisend mit einer leichten Bevorzugung der E-Richtung. Daraus muß geschlossen werden, daß sie nicht gleichzeitig, etwa als Folge eines Sturmes, sondern zu ganz verschiedenen Zeiten umstürzten. Die Stämme fielen auf den Waldboden, d. h. auf Sträucher, Grashorste und totes Holz, die dem Boden im Gegensatz zu einer Hochmooroberfläche so viel Tragfähigkeit verliehen, daß die mit großer Fläche aufliegenden Stämme nur unwesentlich einsinken konnten. Mit einer Ausnahme (Eiche 31) sind alle Bäume an der Oberfläche mehr oder weniger stark abgefault. Die Holzverluste reichen von wenigen $\mathrm{cm}$ bis zum halben Stammdurchmesser. Die oberen Teile dieser Stämme waren also vor ihrer Einbettung im Torf längere Zeit ungeschützt und wurden daher zersetzt (vgl. HAyen 1960).

Da Stubben nur ausnahmsweise beobachtet wurden, ist die Höhenlage, in der der Baum einmal gekeimt und aufgewachsen ist, zunächst nicht sicher feststellbar. An den wenigen sichtbaren Stubben waren die Stämme jedoch sehr dicht über dem Wurzelhals abgebrochen, wie das dem von HAYEN (1960) ausführlich geschilderten und begründeten Normalfall entspricht. Falls wirklich einzelne Stämme höher abgebrochen sein sollten, so dürfte das durch das ja nicht völlig auszuschließende Einsinken der Stämme in die Mooroberfläche wenigstens z. T. wieder ausgeglichen werden. So wird man also sagen dürfen, daß die Stämme im allgemeinen nur wenig über dem Niveau liegen, auf dem die Bäume einmal aufgewachsen sind. Eine Ausnahme von dieser Regel könnte der Stamm 28 sein, der sehr tief an der Basis des Niedermoortorfes in einer Senke liegt. Hier ist nicht mit Sicherheit auszuschließen, daß er hineingefallen oder durch fließendes Wasser dorthin gebracht worden ist. Auch die beiden im Hochmoortorf gefundenen Stämme fügen sich dieser Regel sicher nicht. Da sie mit Sicherheit schon in der Birkenbruchwaldphase abgestorben sind, müssen sie verhältnismäßig lange gestanden und dann höher abgebrochen sein. Wenn sie auch im Hochmoortorf stärker eingesunken sind, so liegen sie immer noch deutlich höher als ihre Stubben.

Diese Befunde sprechen dafür, daß die meisten untersuchten Bäume im Bruchwald aufgewachsen sind. Auch ist das Keimdatum, d. h. die Summe von Absterbedatum und erreichtem Alter der meisten Eichen deutlich jünger als das Alter der ersten Torfbildung (Mitte des 1. vorchristlichen Jahrtausends). Unter den schon frühzeitig abgestorbenen Eichen mögen einige 200- bis 300jährige noch bis in den Mineralboden wurzeln.

\subsubsection{Datierungeines sy chronen Moorhorizontes}

Aus alledem ergibt sich, daß die Stämme bis auf wenige $\mathrm{cm}$ in einer Höhenlage liegen, die ihrem Absterbedatum entspricht. Der Betrag des Höhenwachstums des Moores zwischen Absterbedatum und Falldatum wird durch das geringfügige Einsinken in etwa ausgeglichen. Leider wurde diese Datierungsmöglichkeit für Moorhorizonte zu spät erkannt, so daß nur für einen Teil der Stämme, die zunächst nur mit ihrer Oberkante eingemessen worden waren, der Durchmesser und damit die Höhenlage der Unterkante als datierendes Niveau nachträglich bestimmt werden konnte. Diese Höhen sind mit dem dendrochronologisch bestimmten Alter in Abb. 3 eingetragen.

Die in der Endphase des Niedermoores gehäuften Stämme, die der pollenanalytischen Datierung zufolge kurz vor Chr. Geb. in das Moor geraten sind, zeigen dendrochronologische Alter zwischen 2138 und 2151 v. h. oder umgerechnet 188 bis nach 201 vor Chr. Geb. Sie sind also tatsächlich insgesamt ein wenig älter als die Pollenanalyse erwarten läßt. Sie liegen alle in kurzem Abstand unter oder über dem Niedermoor-Hochmoorkontakt (NHK), der sich so auf kurz nach 2140 v.h. (190 vor Chr. Geb.) 
einengen läßt. Sicherlich ist der Übergang in das Hochmoor auf der $250 \mathrm{~m}$ langen Strecke nicht überall wirklich gleichzeitig vor sich gegangen. Auf kleinen Anhöhen spielte sich dieser Vorgang sicher rascher $\mathrm{ab}$ als in flachen Senken oder Rinnen, die noch länger vom nährstoffreicheren Grundwasser beeinflußt waren. Das zeigt sich am deutlichsten in der Rinne (Bohrung und Eiche 31), wo das 2140 v. h.-Niveau über $40 \mathrm{~cm}$ unter dem Niedermoor-Hochmoorkontakt liegt. Die Rinne war also während der ganzen Niedermoorzeit als deutliche Vertiefung ausgeprägt. In ihr wurde noch einige Zeit Bruchwaldtorf gebildet, als rundum schon die Hochmoorphase erreicht war. Die Tatsache, daß die hier liegende Eiche 31 als einziger der untersuchten Stämme an der Oberseite nicht abgefault ist, zeigt, daß die Auffüllung der Rinne sehr schnell erfolgt sein muß.

\subsubsection{Ableitung der Moorwachstumsgeschwindigkeit}

Einige ältere dendrochronologische Daten und damit datierte Moorhorizonte liegen in deutlich größeren Abständen vom NHK. Es sind die Eichen 41, 28 und 33 mit Sterbealtern zwischen 2190 und nach 2400 v. h. Wenn man die genauen Abstände aller durch Eichen datierten Moorniveaus vom NHK gegen das dendrochronologische Alter aufträgt, auch den des einen Punktes im Hochmoortorf, so ergibt sich eine Kurve, die bis etwa $15 \mathrm{~cm}$ unter dem NHK fast waagerecht verläuft, d. h. in einem Altersbereich zwischen etwa 2135 und 2160 v. h., um dann steil abzufallen bis zu dem Wert der Eiche 28 von frühestens 2400 v. h. und einem Abstand von $46 \mathrm{~cm}$ (Abb. 8). Die Eiche $31 \mathrm{muß}$ in dieser Darstellung natürlicherweise einen weit $a b$ von der Kurve liegenden Punkt ergeben, da sie ja wegen ihrer Rinnenlage aus dem Rahmen dieser einfachen Gesetzmäßigkeit fallen muß.

Aus dieser Kurve läßt sich nun die Wachstumsgeschwindigkeit der Torfe annähernd berechnen. Aus dem rechten, unteren Teil der Kurve, d. h. im Bereich des nährstoffreichen Erlenbruchwald- und Schilftorfes ergibt sich ein Torfzuwachs von etwa $30 \mathrm{~cm}$ in $240 \mathrm{Jah}-$ ren, also rechnerisch ein Wachstum von $0,12 \mathrm{~cm}$ pro Jahr. Im linken, flachen Kurventeil, der etwa dem oberen, nährstoffarmen und sauren Niedermoortorf entspricht, errechnet

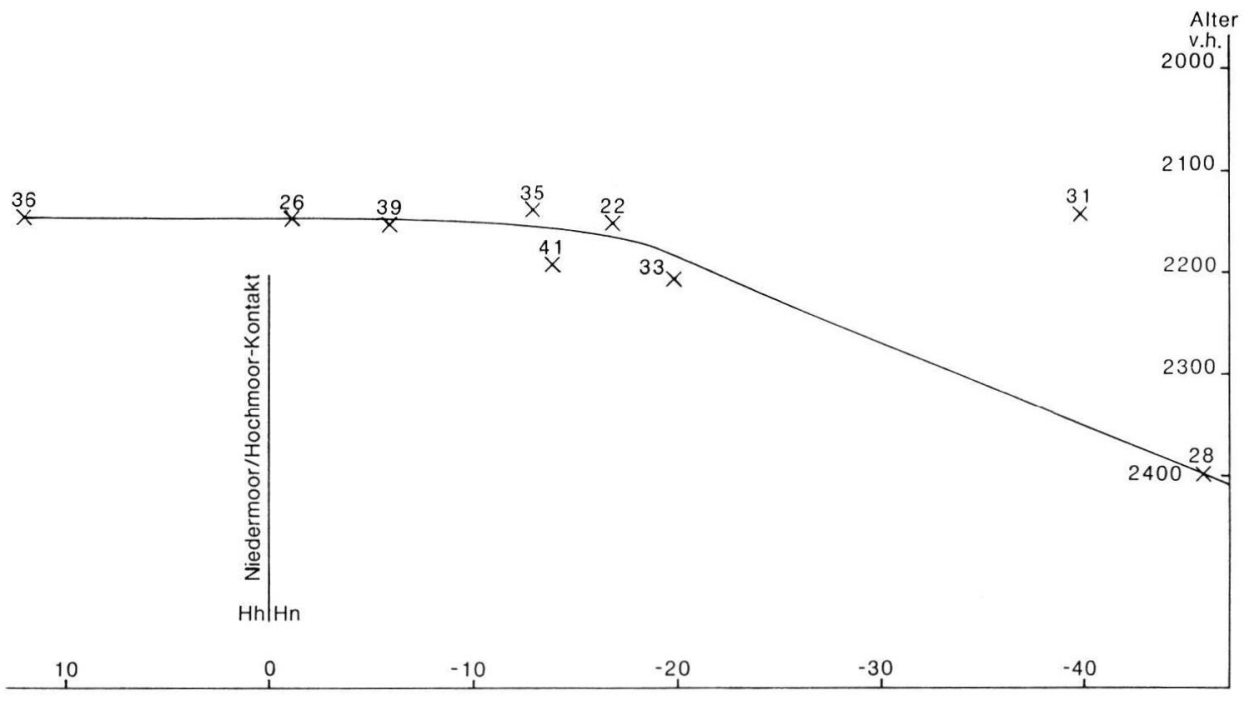

Abb. 8: Abstände der datierten Eichenfunde vom Niedermoor-Hochmoorkontakt (Hochmoor bei Sieden). 
sich bei einem Zuwachs von $28 \mathrm{~cm}$ in nur 25 Jahren eine Wachstumsrate von $1,12 \mathrm{~cm}$ pro Tahr. Wegen der geringen Probenzahl und des infolge Fehlens von Splintresten verbleibenden Datierungsspielraumes kann diese Berechnung nur Überschlagscharakter haben. Dennoch bleibt festzuhalten, daß die Torfbildung sich kurz vor dem Wechsel zum Hochmoor und wohl auch noch kurz danach um ein Mehrfaches rascher vollzogen hat als vorher. Der Übergang von einem nährstoffreichen Niedermoor in ein extrem nährstoffarmes, saures Hochmoor hat sich hier also in wenigen Jahrzehnten vollzogen. Dies Ergebnis stimmt mit einigen wenigen Ergebnissen, die an rezenter Niedermoor- und Hochmoorvegetation gewonnen wurden (BELlamy 1967, J. TüXen, unveröff.), durchaus überein, verdient aber eine Nachprüfung an anderen baumreichen, also Datierungsmöglichkeiten bietenden Mooren.

\subsection{Hinweise auf allgemeine Klimaschwankungen}

Es ist bereits betont worden, daß die Absterbedaten aller hier bearbeiteten Eichen aus dem Borsumer Moor, dem Hochmoor bei Sieden und aus dem Donstorfer Moor überraschenderweise in die Zeitspanne zwischen 2400 und 2100 v. h. fallen (Abb. 5). In Übereinstimmung mit dem von der Entwicklung des Siedener Hochmoores gezeichneten Bild, wo die meisten Eichen in den jüngeren zwei Dritteln dieses Zeitabschnittes gefallen und eingebettet sind, ist auch im Borsumer Moor nur eine einzige Eiche um 2400 v. h. gestorben, alle übrigen erst 100 und mehr Jahre später. Wir engen den Zeitraum des Haupteichensterbens also auf 2300 bis 2100 v. h. ein. Es drängt sich die Frage auf, ob hier nicht überregionale, klimatische Einflüsse wirksam waren, die in allen drei Gebieten gleichzeitig dafür sorgten, daß die Lebensbedingungen für die Eiche in Mooren zu ungünstig wurden. Tatsächlich gibt es viele Hinweise, daß in Hochmooren um diese Zeit Veränderungen ablaufen, die letzten Endes nur großklimatisch gesteuert sein können.

Die Auswertung der in der Literatur angegebenen Daten und ihr Vergleich mit den hier verwendeten Zeitangaben werden allerdings dadurch erschwert, daß bisher nur mit ${ }^{14} \mathrm{C}$ abgesicherte pollenanalytische oder reine ${ }^{14} \mathrm{C}$-Altersbestimmungen vorliegen. Die dadurch bedingten Datierungsspielräume können bewirken, daß trotz gleicher Zeitangaben für bestimmte Ereignisse diese in Wirklichkeit nicht synchron waren. Abweichende Zeitangaben schließen tatsächliche Synchronität nicht unbedingt aus. Allein die Dendrochronologie erlaubt exakte Alterskonnektierungen.

HAYEN (1966) berichtet von einer Häufung der ${ }^{14} \mathrm{C}-$ Daten des SWK (Schwarz-Weißtorfkontakt) zwischen 2050 und $1950 \mathrm{v}$. h. Nach Overвeck (1975) liegen die meisten SWK in Nordwestdeutschland, Schweden und den Britischen Inseln zwischen 2100 und 1850 v. h. mit einer Häufung um 2050 v. h. Die nächst älteren SWK werden erst auf etwa 2500 v. h. datiert. Overbeck hält für „nicht unwahrscheinlich, daß Schwankungen des Niederschlagsnettos der auslösende Faktor für die hydrologischen Veränderungen auf dem Hochmoor gewesen sind“ (1975, p. 598). Die bekannten zeitlichen Verzögerungen des SWK in ein und demselben Moor, die Jahrhunderte betragen können, deutet er als normale Folge einer solchen Klimaänderung. Solche verzögerten SWK sind in den Jahrhunderten zwischen 2500 und 2100 v. h. nicht aufgetreten, die Overbeck für trockener hält.

Die Lebensbedingungen für das ab etwa 2100 v. h. im Siedener Moor aufgewachsene Hochmoor sind also während der letzten, „trockeneren“ Niedermoorphase durch Aufhören des Grundwasseranstieges und die die Verminderung des Nährstoffangebotes an die Vegetation vorbereitet worden. Der gleiche Vorgang hat zum Absterben der Eichen geführt.

Daß sich Schwankungen im Niederschlagsnetto auch in Niedermooren auswirken können, hat erstmalig ScHWAAR (1980) zu zeigen versucht. Er gibt zwei Beispiele aus dem 
hier interessierenden Zeitabschnitt. Um 400 v. Chr. Geb. (2350 v. h.) wird aus dem torfmoosreichen Erlenbruchwald eines Niedermoores in der oberen Wümmeniederung ein torfmoosarmer. Um Chr. Geb. nehmen die Torfmoose wieder zu. Gerade diese Entwicklung zeigt, daß auch hier der Nährstoffhaushalt sich geändert haben mag. Nährstoffarme Erlenbrücher sind im allgemeinen torfmoosreich, nährstoffreiche torfmoosfrei. Hier könnte eine ähnliche Entwicklung wie im Siedener Hochmoor vorliegen. Im Belmer Bruch bei Osnabrück entstand um Chr. Geb. ein Kleinseggenried aus einem Birkenbruchwald, es wurde also feuchter, aber nur in einem Teil des Moores.

Im ganzen besagen diese von SchwaAR beschriebenen Vorgänge, die sich in dem hier interessierenden Zeitraum abgespielt haben, natürlich noch wenig. Man kann gerade in Niedermooren, die von fließendem Wasser abhängig sind, nur schwer ausschließen, daß Anderungen in der Vegetation und damit in der Torfbildung nicht auch oder gar allein von nicht klimatisch bedingten Vorgängen wie etwa der Bibertätigkeit ausgelöst werden. Doch scheint uns die nachgewiesene Gleichzeitigkeit des Eichensterbens, das im Siedener Hochmoor auf Veränderungen des Wasser- und Nährstoffhaushaltes folgte, für eine solche niederschlagsklimatisch bedingte Abhängigkeit auch der Niedermoore zu sprechen.

Eine systematische Erfassung und dendrochronologische Auswertung der in norddeutschen Mooren noch in größerer Zahl zu erwartenden Baumfunde wird in Verbindung mit einer gründlichen geologischen und botanischen Aufnahme der Moorprofile auch zuverlässigere Schlüsse auf Klimaschwankungen zulassen.

\section{Zusammenfassung}

Subfossile Eichenstämme aus dem Borsumer Moor im Emsland, dem Hochmoor bei Sieden und dem Donstorfer Moor wurden jahrringchronologisch untersucht. Dabei erwies sich überraschenderweise die Mehrzahl der Baumstämme aus den drei Mooren als synchron. Sowohl im Borsumer wie im Siedener Moor sind die Eichen innerhalb desselben mehrhundertjährigen Zeitraumes abgestorben. Aus dem Donstorfer Moor steht bislang nur ein Einzelfund zur Verfügung, der in das Ende dieser Phase fällt.

Insgesamt 45 synchrone Eichen wurden zu einer Mittelkurve von 474 Jahren zusammengefaßt. Diese wiederum konnte mit der eisenzeitlichen Auwaldeichenchronologie, aufgebaut aus Stämmen von Main, Fulda, Werra und Oker, überbrückt werden. Dabei ergab sich eine Verlängerung der Eisenzeitchronologie um 215 Jahre zum Allteren. Über eine Häufigkeitsverteilung der Absterbedaten wurde abgeleitet, daß die Eichen aus Mooren im wesentlichen in der Zeit von 350 bis 150 v. Chr. abgestorben sind, wobei sich das Ende dieser Phase bereits mit der Kulmination der Absterbedaten der Fuldaeichen überlappt, während sich die der Auwaldeichen vom Main erst um 75 v. bis 100 n. Chr. häufen.

Jahrringkurvenvergleiche zeigen, daß die Eichen aus norddeutschen Mooren insgesamt inhomogeneren Wachstumsbedingungen ausgesetzt waren als die Eichen aus Auwäldern an Main und Fulda, obwohl letztere sehr viel größere Herkunftsgebiete repräsentieren. Offenbar haben bei den Moorherkünften kleinstandörtliche Unterschiede das Baumwachstum besonders beeinflußt. Dabei müssen die Wuchsbedingungen insgesamt deutlich schlechter gewesen sein als in den süddeutschen Auwäldern, denn die durchschnittliche Ringbreite der dortigen Eichen ist fast doppelt so groß.

Im Hochmoor bei Sieden, dessen Entwicklung mit der Kombination von dendrochronologischen und moorgeologisch-botanischen Methoden untersucht wurde, begann die Moorbildung um 500 v. Chr. Die torfbildende Vegetation war im wesentlichen ein Erlenbruchwald, der unter wechselnden hydrologischen Bedingungen lebte. Kurz vor Chr. Geb. verlangsamte sich der Grundwasseranstieg, die Nährstoffversorgung wurde schlechter, so 
daß aus dem Erlenbruchwald ein Birkenbruch und schließlich ein Hochmoor wurde. Dieser Vorgang brachte alle Bäume des Bruchwaldes zum Absterben. Die Stämme liegen etwa in der Höhenlage, die dem Absterbedatum entspricht. Da die meisten Bäume um 190 v. Chr., der Zeit des Überganges Niedermoor-Hochmoor, abgestorben sind, läßt sich aus der Lage der Stämme die Mooroberfläche für diese Zeit rekonstruieren. Aus den Abständen der datierten Stammlagen im Profil vom Niedermoor-Hochmoorkontakt kann überschlägig die Wachstumsgeschwindigkeit des Moores bestimmt werden. Danach ist das Moor in der Ubergangsphase Niedermoor-Hochmoor um ein Mehrfaches schneller gewachsen als vorher. Dieser Zeitraum hat nur wenige Jahrzehnte gedauert.

Die von uns nachgewiesene Gleichzeitigkeit des Eichensterbens zwischen 350 und 150 v. Chr. in drei weit auseinanderliegenden Mooren drängt die Vermutung auf, daß klimatische Ursachen dafür verantwortlich sind. In vielen Hochmooren hat es zu dieser Zeit einen Wechsel von Schwarztorf zu Weißtorf gegeben, der nach allgemeiner Ansicht von einem Wechsel im Niederschlagsklima gesteuert wird. Aus Niedermooren sind bisher nur wenige Beispiele bekannt, wo womöglich klimatisch bedingte Änderungen der Torfart auftreten. Die hier vorgelegten Untersuchungen machen jedoch wahrscheinlich, daß auch in Niedermooren ein Wechsel von Vegetationstypen und damit von Torfen durch großklimatische Änderungen ausgelöst werden kann.

\section{Schriftenverzeichnis}

Bellamy, D. J. (1967): Some ecological statistics of a "miniature bog". - Oikos, 18 (1): 33-40, 3 Tab., 1 Abb.; Copenhagen.

Delorme, A. (1973): Uber die Bildung von Jahrringbreitenmittelkurven als Grundlage für dendrochronologische Datierungen. - Forstw. Centralblatt, 92: 335-342, 2 Abb.; Verlag Paul Parey, Hamburg und Berlin.

- (1978): Fortschritte beim Aufbau der Göttinger Eichenjahrringchronologie des Postglazials. Neue Ausgrab. u. Forsch. in Nieders., 12: 243-246, 1 Abb., 1 Tab.; Hildesheim.

- (1979): Die dendrochronologischen Methoden. - Allgem. Forst-Zeitschrift, 49: 1345-1347; München.

ElLING, W. (1966): Untersuchungen über das Jahrringverhalten der Schwarzerle. - FLORA, Abt. B, 156: 155-201, 17 Abb., 4 Tab.; Jena.

Gerh, M. A. (1971): Die Anwendung der 14C-Methode. - Clausthaler Tektonische Hefte: 11, 118 S., 12 Abb., 5 Tab.; Clausthal-Zellerfeld.

HAYEN, H. (1960): Erhaltungsformen der in den Mooren gefundenen Baumreste. - Oldenburger Jb., 59, Teil 2: 21-49, 15 Abb.; Oldenburg.

- (1966): Moorbotanische Untersuchungen zum Verlauf des Niederschlagsklimas und seiner Verknüpfung mit der menschlichen Siedlungstätigkeit. - Neue Ausgrab. u. Forsch. in Niedersachsen, 3: 280-307, 14 Abb.; Hildesheim.

Hesemann, J. (1950): Uber die stratigraphische Stellung der großen Emsterrasse im Münsterland. — Geol. Jb., 64: 633-641, 2 Abb., 2 Tab.; Hannover.

Huber, B. (1943): Über die Sicherheit jahrringchronologischer Datierung. - Holz als Roh- und Werkstoff, 6, H. 10/12: 263-268, 7 Abb., 4 Tab.; Berlin.

- (1969): Dendrochronologie. - Handbuch der Mikroskopie in der Technik: S. 171-211, Umschau-Verlag, Frankfurt/Main.

MeYer, K. D. (1977): Erläuterungen zu Blatt 3610, Salzbergen. - Geolog. Kt. Niedersachsen $1: 25$ 000: 111 S., 5 Tab., 1 Taf., 3 Kt.; Hannover.

MüLleR, H. (1956): Ein Beitrag zur holozänen Emstalentwicklung zwischen Meppen und Dörpen auf Grund von pollenanalytischen Untersuchungen. - Geol. Jb., 71: 491-504, 6 Abb., 1 Tab.; Hannover. 
Munaut, A. V. (1967): Etude paléo-écologique d'un gisement tourbeux situé â Terneuzen (PaysBas). - Ber. v. d. Rijksdienst v. h. Oudheidkundig Bodemonderzoek, 17: 7-27, 15 Abb.; Amersfoort.

- \& Casparie, w. a. (1971): Etude dendrochronologique des Pinus sylvestris L. subfossiles provenant de la tourbière d'Emmen (Drenthe, Pays-Bas). - Rev. of Paleobt. and Palynol.: 11, 201-226, 8 Abb., 14 Tab.; Amsterdam.

Overbeck, F. (1975): Botanisch-geologische Moorkunde unter besonderer Berücksichtigung Nordwestdeutschlands: 719 S., 38 Tab., 263 Abb.; Neumünster (Wachholzverlag).

Pilcher, J. R., Hillam, J., Baillie, M. G. L. \& Pearson, G. W. (1977): A long sub-fossil oak treering chronology from the north of Ireland. - New Phytol.: 79: 713-729, 9 Abb.; Oxford.

Schмidt, B. (1977): Der Aufbau von Jahrringchronologien im Holozän mit Eichen (Quercus sp.) aus dem Rhein-, Weser- und Werragebiet. - In: Frenzel, B. (ed): Dendrochronologie und postglaziale Klimaschwankungen in Europa. - Erdwiss. Forsch., 13: 91-98, 3 Abb.; Wiesbaden.

Schneekloth, H. \& Schneider, S. (1970): Die Moore in Niedersachsen. 1. Teil. Bereich des Blattes Hannover der Geologischen Karte der Bundesrepublik Deutschland (1:200 000). Veröff. Nieders. Inst. f. Landeskde u. Landesentw. a. d. Univ. Göttingen. Reihe A. 1. 96 (1): 60 S., 1 Kt.; Göttingen.

SchwaAr, J. (1980): Sind die hygro- und xeroklinen Phasen der Hochmoorbildung (Overbeck) und bestimmte Phasen der Niedermoorbildung synchrone Vorgänge gleicher Ursache? Ein Beitrag zu einem wenig beachteten Problem. - In: R. Tüxen (Herausg.), Epharmonie. Ber. Internat. Symp. Internat. Verein. f. Vegetationskde: 95-119, 12 Abb.; Vaduz.

Thiermann, A. (1963): (1973), mit Beiträgen von Dubber, H.-J., Kalterherberg, J., Koch, M. \& Rehagen, H.-W.: Erläuterungen zu Blatt 3710, Rheine. - Geol. Kt. Nordrh.-Westf. $1: 25000: 174$ S., 16 Abb., 12 Tab., 5 Taf.; Krefeld.

- (1975), mit Beiträgen von Braun, F. J., Kalterherberg, J., Rehagen, H.-W., Suchan, K.-H., Will, K.-H. \& Wolburg, J.: Erläuterungen zu Blatt 3610 Hopsten. - Geol. Kt. Nordrh.Westf. $1: 25$ 000: 214 S., 21 Abb., 9 Tab., 5 Taf.; Krefeld.

TüxEN, J. (1967): Naturschutzgebiet „Duvenstedter Brook“, Vegetationstypen. - Schr.reihe Landesstelle f. Naturschutz u. Landschaftspflege d. Freien u. Hansestadt Hamburg, 1: 120 S., 24 Abb., 33 Tab., 4 Kt.; Hamburg.

Manuskript eingegangen am 4. 5. 1981.

$\mathrm{Nachtrag}(18.8 .81)$

Gestützt auf jüngst von BECKER veröffentlichte Ergebnisse (Fällungsdaten römischer Bauhölzer anhand einer 2350jährigen süddeutschen Eichen-Jahrringchronologie. - Fundberichte aus BadenWürttemberg 6: 369-386, 9 Abb., 8 Tab., o. O.; 1981) konnte inzwischen die Eiszeitliche Chronologie mit der Göttinger Auwaldeichenchronologie für das Frühmittelalter zu einer von 573 v. Chr. bis $785 \mathrm{n}$. Chr. reichenden Absolutchronologie verknüpft werden. Alle hier mit einer Streuung von \pm 23 Jahren angegebenen gemittelten 14-D-Daten lassen sich infolgedessen im Zeitraum 2594-2022 v. h. durch Subtraktion von 2021 Jahren auf wirkliche Kalenderjahre v. Chr., bzw. im Zeitraum 2021-1784 v. h. durch Subtraktion von 2022 Jahren auf wirkliche Kalenderjahre n. Chr. umrechnen.

Die Hauptphase des Absterbens von Eichenbeständen in den drei Mooren, zunächst über 14-C in die Zeit von 350-150 v. Chr. datiert, kann nunmehr sicher mit 280-80 v. Chr. angegeben werden. 